\title{
Etude comparée du comportement alimentaire de deux poissons cavernicoles (Anoptichthys jordani Hubbs et Innes et Caecobarbus geertsi Blgr.)
}

\author{
par \\ Georges THINÈS et Nicole WISSOCQ*
}

\section{INTRODUCTION}

Dans un travail préliminaire paru dans ce même Journal, Thinès, Soffié et Vandenbussche (1966) avaient procédé à l'analyse du comportement alimentaire du poisson cavernicole Anoptichthys Gen. et des hybrides $\mathrm{F}_{1}$ obtenus par croisement Astyanax x Anoptichthys, la première espèce étant la forme ancestrale épigée de la seconde. Les résultats de ces recherches avaient mis en évidence les traits essentiels de la réaction de la forme cavernicole Anoptichthys à la stimulation chimique. Ces données avaient également permis de caractériser la stéréotypie d'exploration de ce poisson consécutive à la stimulation chimique initiale. Les auteurs précités avaient établi que l'exploration alimentaire s'effectuait sous la forme d'une plongée vers le fond suivie d'une recherche active localisée autour du point d'impact de la nourriture sur le substrat. L'efficacité de saisie, comparée à celle de la forme ancestrale Astyanax, est très déficitaire. De plus, la plongée vers le fond peut être déclenchée par des stimuli perturbants de nature mécanique. L'ensemble de ces observations avait été soumis à la discussion et avait amené à conclure que le comportement alimentaire, outre les déficits évidents qu'il accuse sur le plan de l'efficacité spatio-temporelle de saisie, intervenait à titre substitutif dans des situations déclenchant des comportements phobiques typiques chez la forme épigée Astyanax.

\section{POSITION DU PROBLEME}

Si l'on se réfère à la classification de Vandel (1964), l'Anoptichthys est un cavernicole récent, tandis que le Caecobarbus est un cavernicole ancien. Cette différence d'ancienneté phylogénique justifiait à elle seule une comparaison systématique du comportement alimentaire des deux espèces. Celles-ci appartenant l'une à la famille des Characidae, l'autre à la famille des Cyprinidae, toutes deux rangées dans l'ordre des Cypriniformes, la comparaison s'imposait également du point de vue systématique.

L'observation du comportement respectif des deux espèces dans des conditions identiques révèle à la fois des similitudes et des différences. Des descriptions antérieures (Lüling, 1954 - Thinès, Soffié et Vandenbussche, 1966) avaient montré

* Centre de Psychologie expérimentale et comparée, Université de Louvain 3041 Pellenberg, Belgique. 
que l'Anoptichthys manifeste une polarisation très nette sur le fond du milieu qu'il occupe. Ce caractère de l'exploration alimentaire est tellement typique, que nous avons pu l'utiliser antérieurement comme indice de conditionnement pour étudier les réactions de l'espèce $A$. jordani Hubbs et Innes à différentes radiations chromatiques (Thinès et Kähling, 1957). Ce trait comportemental semble lié à la disparition de la coordination visuelle - motrice consécutive à la régression oculaire. Heuts et Thinès (1971) ont observé l'apparition assez inattendue d'une exploration polarisée sur le substrat chez des Barbus conchonius Hamilton accidentellement aveuglés et dépigmentés à la suite d'un traitement au mercurochrome, les témoins manifestant au contraire une tendance initiale à nager vers le surface. Des résultats semblables ont été obtenus par Thinès et Capon (1971) sur Danio malabaricus. Glaser (1968) a également observé que l'Anoptichthys découvre plus rapidement sa nourriture que des espèces aveuglées observées dans les mêmes conditions (Phoxinus phoxinus, Hemigrammus caudovittatus et Gasterosteus aculeatus). Le même auteur remarque une tendance à s'orienter vers le substrat chez les Phoxinus aveuglés. Chez le Caecobarbus, la tendance à nager en direction du substrat après la stimulation chimique existe également, mais elle semble être associée de façon moins rigide à l'exploration alimentaire que chez l'Anoptichthys. Les observation très nombreuses que nous avons pratiquées sur ces poissons cavernicoles remarquables, montrent pourtant qu'ils sont capables de poursuivre des proies inanimées de dimensions variables au cours du trajet couvert par celles-ci entre la surface et le substrat. Cependant, les poissons frôlent la plupart du temps les proies sans s'orienter vers celles-ci et ils ne parviennent à les saisir que très rarement. Lorsque la nourriture a atteint le fond, la localisation et la saisie de la proie sont plus lentes et plus imprécises que chez l'Anoptichthys.

Les expériences à effectuer ultérieurement sur le comportement alimentaire de ces poissons cavernicoles devaient dès lors prendre avant tout en considération le niveau vertical de l'exploration dans des conditions rigoureusement contrôlées. Les résultats obtenus par Heuts et Thinès (1971) et par Thinès et Capon (1971) avaient montré que la disparition du contrôle visuel peut modifier à court terme la répartition en hauteur de l'exploration alimentaire d'une espèce épigée. On pouvait donc supposer que la labilité comportementale mise en évidence dans le cas des $B$. conchonius aveuglés pourrait se manifester chez les deux espèces cavernicoles aveugles dans des conditions appropriées de stimulation.

C'est pourquoi il fut décidé de procéder à des expériences dans lesquelles les réactions des poissons seraient évaluées en utilisant la technique de Heuts et Thinès (1971), dans laquelle les positions des animaux sont repérées en hauteur dans différentes conditions de stimulation. En vue de créer deux types nettement distincts de conditions, nous avons utilisé deux espèces de proies, l'une constituée de Tubifex, l'autre de Daphnies sèches. Lorsqu'on introduit la première dans l'aquarium expérimental, celle-ci descend immédiatement sur le fond; quant à la seconde, elle flotte en surface et ne pénètre qu'exceptionnellement dans la masse liquide. Il était donc aisé d'observer et de mesurer les réactions des poissons après une période prolongée de nutrition utilisant exclusivement l'un ou l'autre type de proie. Dans nos expériences, les groupes de poissons utilisés avaient été nourris avec 
des Tubifex ou des Daphnies sèches une fois par jour pendant les 8 jours précédant les observations définitives.

\section{MATERIEL ET METHODES}

Les expériences ont été effectuées dans les mêmes conditions sur des groupes de 6 à 10 individus adultes et sur les mêmes individus isolés. Les poissons ont été observés dans 3 aquariums de $60 \times 30 \times 40 \mathrm{~cm}$ dans lesquels la hauteur de l'eau était de $32 \mathrm{~cm}$. Dans toutes les expériences, l'eau a été maintenue à une température constante de $24^{\circ} \mathrm{C}$ et le local expérimental a été soumis à un cycle L D: $12 / 12$, l'éclairement diffus pendant la période L étant d'environ 250 Lux. Les poissons purent s'adapter à l'aquarium dans lequel ils vivaient pendant 1 semaine et aucun animal ne fut transféré d'un aquarium à l'autre au cours des observations, sauf bien entendu dans le cas des observations sur individus isolés. Dans ces dernières conditions, les animaux hors expériences étaient placés dans un autre aquarium identique à l'aquarium expérimental. Lors de la reconstitution du groupe, une nouvelle semaine d'adaption précédait chaque fois les observations ultérieures.

Les aquariums étaient divisés en 3 zones d'observation superposées, par deux traits horizontaux tracés au crayon marqueur sur le verre aux niveaux de $10 \mathrm{~cm}$ et de $20 \mathrm{~cm}$. Pour obtenir des données quantitatives, il suffisait de compter le nombre d'animaux présents dans 2 des 3 zones, le nombre d'animaux présents dans la 3ème étant obtenu par soustraction. Chaque séance d'observation durait 15 minutes. Pendant les 5 premières minutes, la position du poisson isolé ou la distribution du groupe était relevée toutes les 10 secondes. Le stimulus était présenté au terme de cette période dite AS (Avant la stimulation) et les relevés continuaient toutes les 10 secondes pendant 10 minutes. Cette seconde période, dite PS, est divisée pour les besoins de l'analyse en deux sous-périodes successives de 5 minutes, dites respectivement $\mathrm{PS}_{1}$ et $\mathrm{PS}_{2}$. La disposition spatiale des zones d'observation et la répartition temporelle d'une séance complète sont schématisées à la Fig. 1 .

Ce schéma a été utilisé dans la représentation graphique de tous les résultats. Dans les observations effectuées sur les individus isolés, la représentation graphique se réduit évidemment à celle des fluctuations dans le temps d'un point unique aux 3 niveaux. Les symboles utilisés $\left(\mathrm{AS}, \mathrm{PS}_{1}, \mathrm{PS}_{2}\right)$ peuvent être affectés d'un indice supplémentaire indiquant le niveau correspondant à l'une des zones d'observation. Ainsi $\mathrm{AS}_{\mathrm{s}}=$ période avant stimulation, niveau supérieur, $\mathrm{PS}_{2 \mathrm{i}}=$ période poststimulus, sous-période 2, niveau inférieur, etc. Chaque type d'observation a été répété 4 fois à des intervalles de plusieurs jours afin d'éliminer les effets des observations antérieures. Au rythme de 1 relevé toutes les 10 secondes, on obtient 30 observations momentanées pour chaque période $\mathrm{AS}, \mathrm{PS}_{1}$ et $\mathrm{PS}_{2}$. La variable étudiée étant la distribution verticale, le moment de la saisie effective de la nourriture n'a pas été pris en considération dans l'analyse des résultats. Etant donné que dans deux situations expérimentales types, on utilisait soit de la nourriture descendant par gravité (Tubifex), soit de la nourriture flottante (Daphnies), les moyennes des observations par période de 5 minutes ont été calculées uniquement 


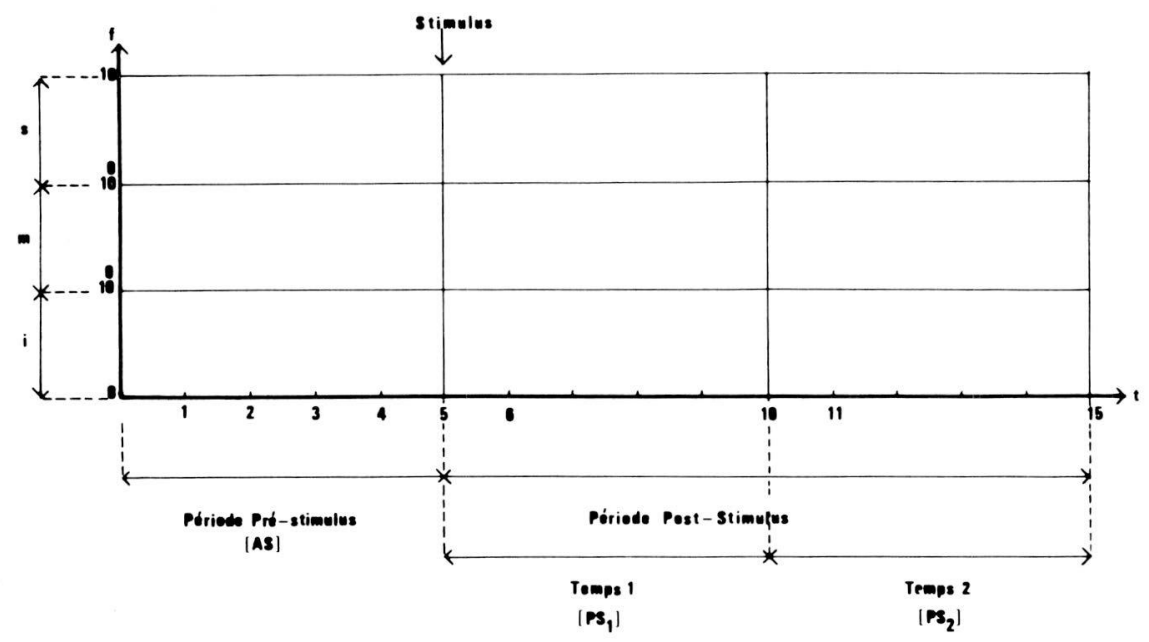

Fig. 1. Schéma spatio-temporel des observations. En abscisse, temps d'observation $t$ en minutes. En ordonnée, niveaux verticaux d'observation en $\mathrm{cm}$ auxquels les fréquences f sont relevées. Les lettres $\mathrm{i}, \mathrm{m}$ et $\mathrm{s}$ désignent les niveaux respectifs inférieur, moyen et supérieur. Explications complémentaires dans le texte.

pour le niveau supérieur et pour le niveau inférieur, les données relatives au niveau moyen figurent seulement dans les graphiques. Pour établir si les différences entre moyennes supérieures et les moyennes inférieures étaient significatives ou non, nous avons utilisé l'épreuve U (Mann-Whitney). Outre le Tubifex et la nourriture flottante, nous avons également utilisé comme stimulus un choc mécanique appliqué à la paroi de face de l'aquarium, selon le procédé de Heuts et Thinès (1971) et de Thinès et Capon (1971).

Dans chacune des 3 situations-types (Tubifex, nourriture flottante, choc mécanique), nous avons utilisé 2 groupes de 10 Anoptichthys et 2 groupes de 6 Ceacobarbus, chacun des 2 groupes d'Anoptichthys et de Caecobarbus ayant été nourri avec un type exclusif de nourriture au cours de la semaine initiale d'adaptation. Les expériences dans lesquelles on stimulait les poissons au moyen d'un choc mécanique, ont été effectuées ultérieurement sur chacun des deux groupes précités d'Anoptichthys et de Caecobarbus. Les observations individuelles ont précédé, dans tous les cas, les observations sur les groupes. L'analyse statistique a été effectuée sur les données numériques individuelles et collectives obtenues à l'intérieur de chaque groupe de poissons non seulement au point de vue des niveaux, mais aussi pour chaque niveau, au point de vue de la période $\left(\mathrm{AS}, \mathrm{PS}_{1}\right.$, $\mathrm{PS}_{2}$ ). Les groupes utilisés ont également été comparés 2 à 2 au point de vue du stimulus utilisé. Les résultats de cette dernière analyse ne sont pas donnés en détail et n'interviennent que dans la discussion finale. 
RESULTATS

\section{I - RESULTATS OBTENUS SUR ANOPTICHTHYS JORDANI.}

\section{A. Description des comportements}

\section{Période antérieure à l'intervention du stimulus (AS)}

Les résultats obtenus au cours de la période AS sont très semblables chez les 10 Anoptichthys observés individuellement et nourris avec des Tubifex. Les poissons

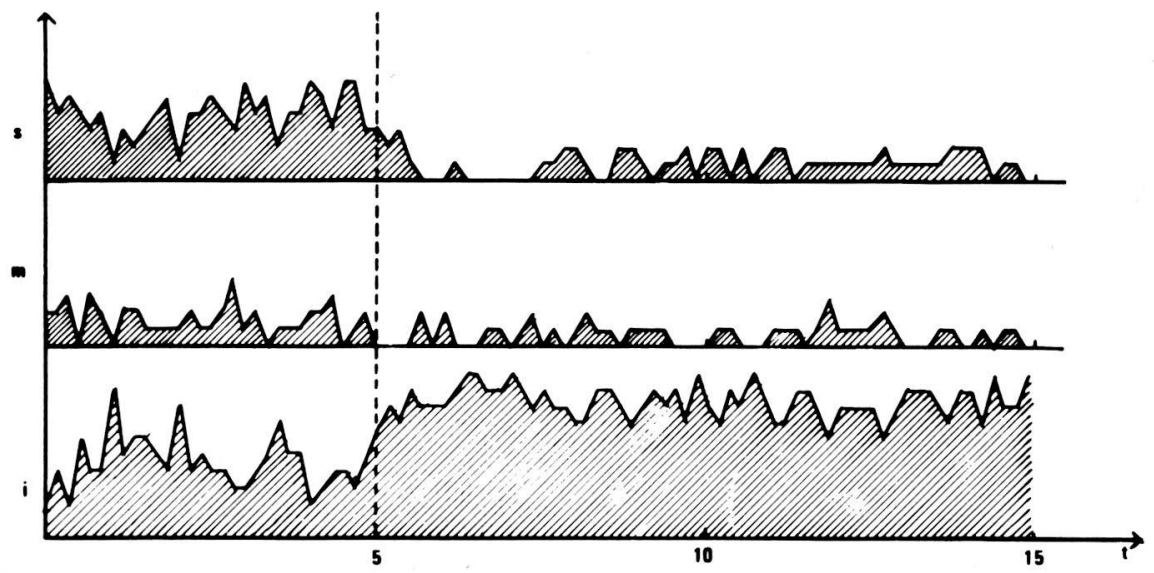

Fig. 2a. Distribution verticale caractéristique d'un groupe de 10 Anoptichthys nourris antérieurement et stimulés avec des Tubifex. Abscisses: temps en minutes. Ordonnées: nombre de poissons (f) en valeur absolue. i,m,s: niveaux respectifs inférieur, moyen et supérieur. Le trait vertical en pointillé indique le moment d'intervention du stimulus.

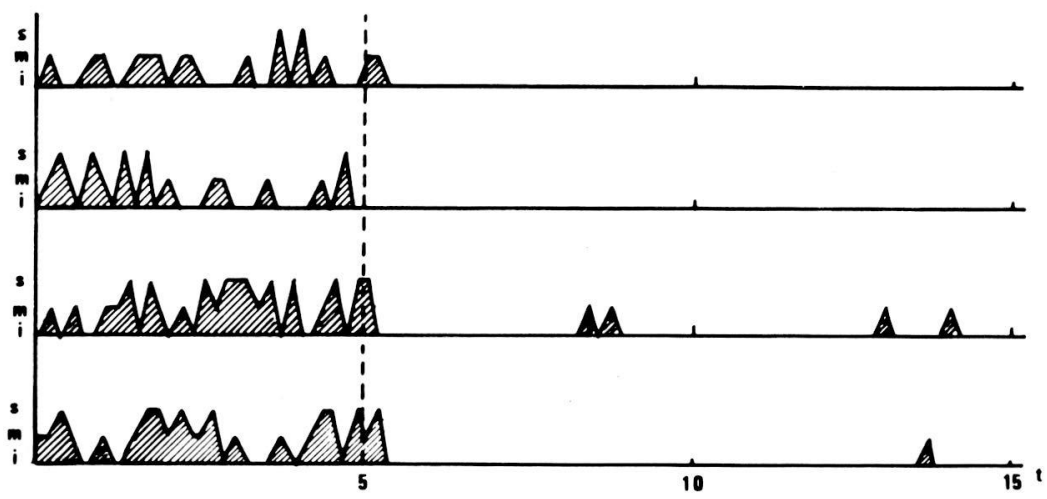

Fig. 2b. Distribution caractéristique des positions verticales d'un Anoptichthys observé individuellement (nourri antérieurement et stimulé avec des Tubifex). Relevé de 4 expériences identiques. Mêmes indications qu'en (a). 
répartissent irrégulièrement leur activité aux trois niveaux de l'aquarium. Il en va de même chez les 10 poissons observés individuellement et nourris avec de la nourriture flottante. On note toutefois que les poissons nourris avec des Tubifex ont tendance à se répartir préférentiellement aux niveaux moyen et inférieur, tandis que les poissons nourris avec de la nourriture flottante occupent plutôt les niveaux moyen et supérieur. Les caractéristiques relevées sont légèrement plus accentuées chez les poissons observés en groupe.

\section{Période postérieure à l'intervention du stimulus $\left(P S_{1}+P S_{2}\right)$}

Tant chez les individus isolés que dans les groupes, l'introduction de Tubifex dans l'aquarium provoque une accélération de la nage et une plongée vers le substrat, où les animaux se livrent à une exploration active en quête de nourriture. Ce comportement s'observe tant chez les poissons en groupe que chez les poissons isolés qui ont été nourris antérieurement avec des Tubifex. Aux cours de $\mathrm{PS}_{2}$, l'exploration du substrat se maintient, une tendance au rétablissement de la distribution initiale s'amorçant toutefois entre la 8ème et la 15ème minute.

En ce qui concerne les effets de la nourriture flottante, celle-ci détermine une accélération identique de la nage chez les animaux nourris antérieurement avec des Daphnies. Toutefois l'exploration n'est dirigée vers la surface que pendant une durée de 1 à 6 minutes. Au terme de cette période, les animaux se concentrent à nouveau au niveau inférieur. Les poissons se maintiennent toutefois plus longtemps au niveau supérieur (envison 10 minutes, entrecoupées de quelques rares descentes vers le niveau inférieur).

L'intervention d'un choc mécanique sur la paroi de l'aquarium exerce un effet très différent. Les réactions observées sont avant tout des comportement phobiques, les poissons nageant dans toutes les directions sans manifester de signes d'exploration typiques. Chez les Anoptichthys nourris avec des Tubifex, les individus rétablissent leur répartition initiale vers la 4ème minute de $\mathrm{PS}_{2}$ en sorte que la tendance à nager au niveau inférieur réapparaît à ce moment. Les individus nourris avec des Daphnies ont tendance à se porter au niveau supérieur. Les réactions diffèrent dans les groupes et chez les individus isolés dans le cas des poissons nourris antérieurement avec des Tubifex.

L'observation des comportements des Anoptichthys révèle donc les faits essentiels suivants:

1. Les stimuli chimiques et le stimulus mécanique provoquent tous une accélération de la nage.

2. Seuls les stimuli chimiques provoquent une accélération de la nage suivie d'exploration active.

3. Le stimulus mécanique provoque une accélération de la nage suivie immédiatement d'un comportement de fuite.

4. Les faits observés concordent avec l'analyse antérieure de Thinès, Soffié et Vandenbussche (1966) pour ce qui concerne les phases successives de chémoréception et d'exploration.

5. Le niveau préférentiel d'exploration est déterminé par le type de nourriture 
utilisé comme stimulus: niveau inférieur pour les poissons nourris antérieurement avec des Tubifex et stimulés avec ceux-ci; niveau supérieur pour les poissons nourris antérieurement avec de la nourriture flottante et stimulés avec celle-ci.

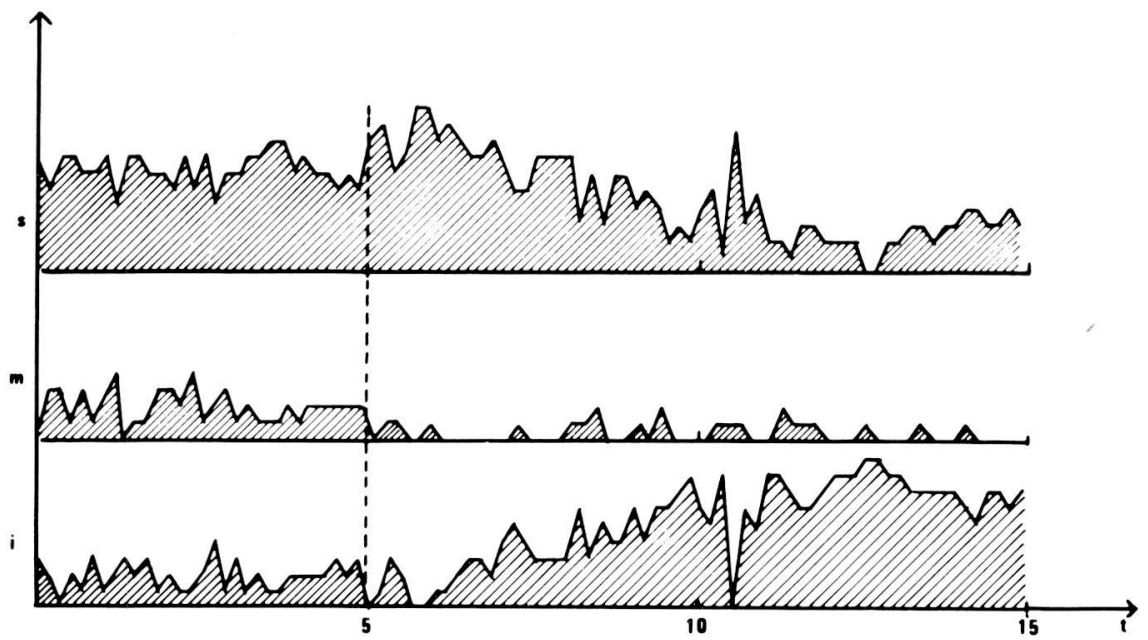

Fig. 3a. Distribution verticale caractéristique d'un groupe de 10 Anoptichthys nourris antérieurement et stimulés avec de la nourriture flottante. Abscisses: temps en minutes. Ordonnées: nombre de poissons (f) en valeur absolue. i,m,s: niveaux respectifs inférieur, moyen et supérieur. Le trait vertical en pointillé indique le moment d'intervention du stimulus.

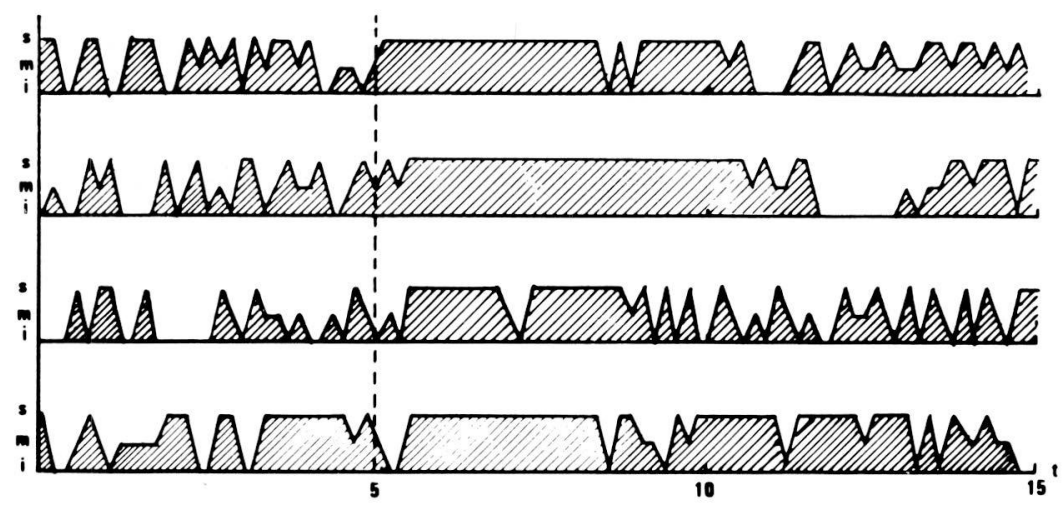

Fig. 3b. Distribution caractéristique des positions verticales d'un Anoptichthys observé individuellement (nourri antérieurement et stimulé avec de la nourriture flottante). Relevé de 4 expériences identiques. Mêmes indications qu'en (a). 


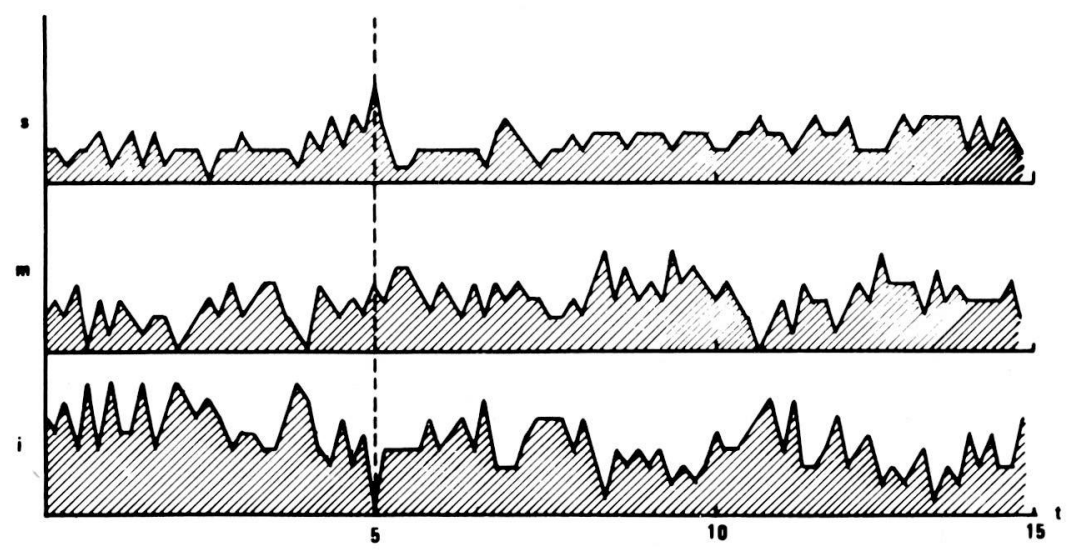

Fig. 4a. Distribution verticale caractéristique d'un groupe de 10 Anoptichthys nourris antérieurement avec des Tubifex et stimulés par un choc mécanique. Abscisses: temps en minutes. Ordonnées: nombre de poissons (f) en valeur absolue. i,m,s: niveaux respectifs inférieur, moyen et supérieur. Le trait vertical en pointillé indique le moment d'intervention du stimulus.

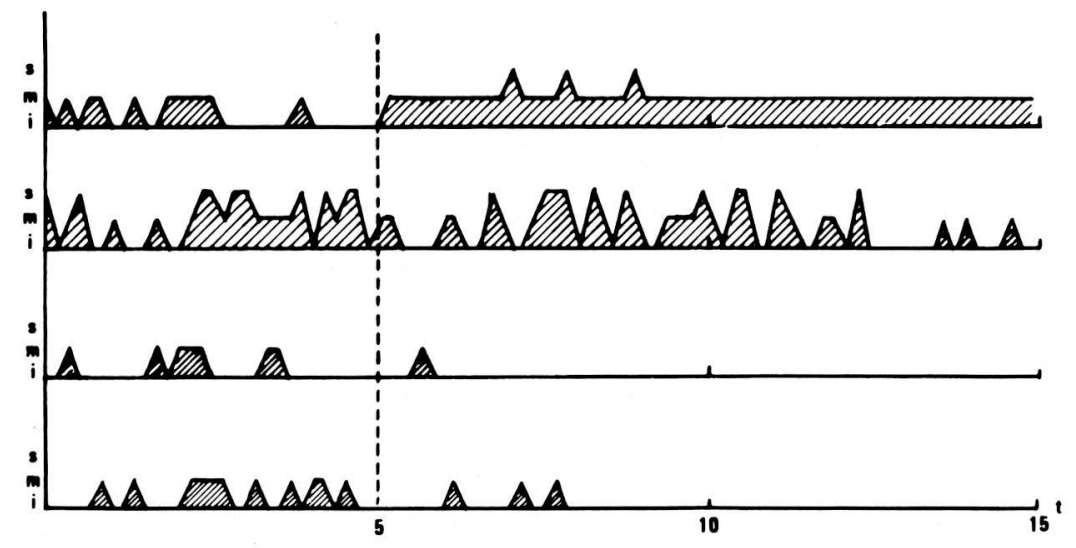

Fig. 4b. Distribution caractéristique des positions verticales d'un Anoptichthys observé individuellement (nourri antérieurement avec des Tubifex et stimulé par un choc mécanique). Relevé de 4 experiences identiques. Mêmes indications qu'en (a). 


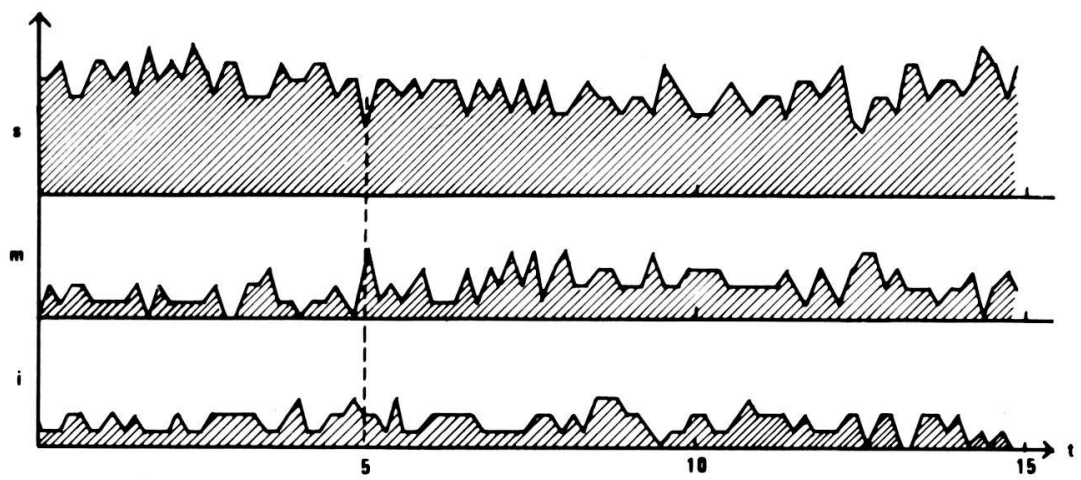

Fig. 5a. Distribution verticale caractéristique d'un group de 10 Anoptichthys nourris antérieurement avec de la nourriture flottante et stimulés par un choc mécanique. Abscisses: temps en minutes. Ordonnées: nombre de poissons (f) en valeur absolue. $\mathrm{i}, \mathrm{m}, \mathrm{s}$; niveaux respectifs inférieur, moyen et supérieur. Le trait vertical en pointillé indique le moment d'intervention du stimulus.

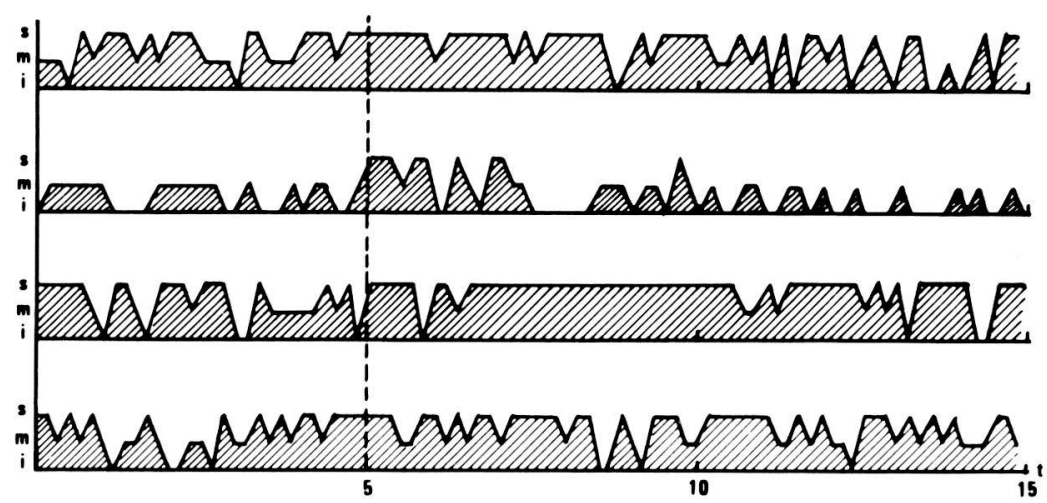

Fig. 5b. Distribution caractéristique des positions verticales d'un Anoptichthys observé individuellement (nourri antérieurement avec de la nourriture flottante et stimulé par un choc mécanique). Relevé de 4 expériences identiques. Mêmes indications qu’en (a).

\section{B. Analyse statistique des résultats}

L'analyse statistique des résultats obtenus sur Anoptichthys a été effectuée à partir des médians des observations. Comme nous l'avons signalé antérieurement, les niveaux de signification ont été calculés au moyen de l'épreuve U de Mann-Whitney $(\alpha=0,05)$. Les moyennes sont données au tableau 1 pour les Anoptichthys observés en groupe et au tableau 2 pour les Anoptichthys observés individuellement. 


\section{Tableau 1}

Moyennes des observations effectuées sur Anoptichthys jordani dans les diverses conditions expérimentales. Poissons observés en groupe.

\begin{tabular}{|c|c|c|c|c|c|c|c|c|c|c|c|c|}
\hline \multirow{2}{*}{ séance } & \multirow{2}{*}{$\begin{array}{l}\text { Sti. } \\
\text { Pér. } \\
\text { Niv. }\end{array}$} & \multicolumn{2}{|c|}{$\begin{array}{l}\text { Nourris \& Sti. } \\
\text { avec Tubifex }\end{array}$} & \multicolumn{3}{|c|}{$\begin{array}{l}\text { Nourr. Tub. } \\
\text { stim. Choc }\end{array}$} & \multicolumn{3}{|c|}{$\begin{array}{l}\text { Nourr. flott. } \\
\text { Stim. flott. }\end{array}$} & \multicolumn{3}{|c|}{$\begin{array}{l}\text { Nourr. flott. } \\
\text { stim. Choc }\end{array}$} \\
\hline & & AS P & $1 \mathrm{PS}_{2}$ & AS & $\mathrm{PS}_{1}$ & $\mathrm{PS}_{2}$ & AS & $\mathrm{PS}_{1}$ & $\mathrm{PS}_{2}$ & AS & $\mathrm{PS}_{1}$ & $\mathrm{PS}_{2}$ \\
\hline \multirow{2}{*}{ I } & $\mathrm{S}$ & 3 & 0 & 2 & 2 & 3 & 6 & 6,5 & 4 & 7 & 6 & 6 \\
\hline & $\mathrm{i}$ & $\begin{array}{ll}3 & 10\end{array}$ & 10 & 5,5 & 4 & 5 & 2 & 3 & 7 & 2 & 2 & 2 \\
\hline \multirow{2}{*}{ II } & $\mathrm{s}$ & 20 & 1 & 3 & 3 & 2 & 6 & 7 & 3,5 & 6 & 5 & 5 \\
\hline & $\mathrm{i}$ & $\begin{array}{ll}5 & 10\end{array}$ & 9 & 5 & 5 & 5 & 2 & 3 & 6,6 & 6 & 3 & 2 \\
\hline \multirow{2}{*}{ III } & $\mathrm{s}$ & 20 & 0 & 2 & 2 & 1 & 6 & 6 & 2 & 7 & 6 & 6 \\
\hline & $\mathrm{i}$ & $\begin{array}{ll}7 & 10\end{array}$ & 9 & 4 & 4 & 4 & 2 & 4 & 6 & 2 & 2 & 2 \\
\hline \multirow{2}{*}{ IV } & $\mathrm{s}$ & $\begin{array}{ll}4 & 1\end{array}$ & 0 & 2 & 2 & 2 & 6 & 6 & 2,5 & 5 & 4,5 & 7 \\
\hline & i & 4 & 8 & 5 & 4 & 5 & 2 & 3,5 & 5 & 2 & 3 & 2 \\
\hline
\end{tabular}

\section{Tableau 2}

Moyennes des observations effectuées sur Anoptichthys jordani dans les diverses conditions expérimentales. Poissons observés individuellement.

\begin{tabular}{|c|c|c|c|c|c|c|c|c|c|c|c|c|c|}
\hline \multirow[t]{2}{*}{ séance } & \multirow{2}{*}{$\begin{array}{l}\text { Sti. } \\
- \\
\text { Pér. } \\
\text { Niv. }\end{array}$} & \multicolumn{3}{|c|}{$\begin{array}{l}\text { Nourris \& Sti. } \\
\text { avec Tubifex }\end{array}$} & \multicolumn{3}{|c|}{$\begin{array}{l}\text { Nourri. Tub. } \\
\text { stim. Choc }\end{array}$} & \multicolumn{3}{|c|}{$\begin{array}{l}\text { Nourri. flott. } \\
\text { stim. flott. }\end{array}$} & \multicolumn{3}{|c|}{$\begin{array}{l}\text { Nourri. flott. } \\
\text { stim. Choc }\end{array}$} \\
\hline & & AS & $\mathrm{PS}$ & $\mathrm{PS}_{2}$ & AS & $\mathrm{PS}_{1}$ & $\mathrm{PS}_{2}$ & AS & $\mathrm{PS}_{1}$ & $\mathrm{PS}_{2}$ & AS & $\mathrm{PS}_{1}$ & $\mathrm{PS}_{2}$ \\
\hline \multirow{2}{*}{ I } & $\mathrm{s}$ & 4 & 0 & 1 & 4 & 3 & 5 & 4 & 7 & 4 & 4 & 6 & 4,5 \\
\hline & i & 9 & 9 & 8 & 3,5 & 3 & 4 & 3 & 2 & 4 & 2 & 1 & 1 \\
\hline \multirow{2}{*}{ II } & $\mathrm{s}$ & 4 & 1 & 0 & 3 & 3 & 2,5 & 4 & 7 & 6 & 4,5 & 4,5 & 4 \\
\hline & $\mathrm{i}$ & 4 & 8 & 10 & 4,5 & 3 & 2,5 & 3 & 2 & 3 & 3 & 2 & 2,5 \\
\hline \multirow{2}{*}{ III } & $\mathrm{s}$ & 2,5 & 1 & 0 & 5 & 3 & 3,5 & 4 & 6 & 7 & 4 & 5 & 4 \\
\hline & $\mathrm{i}$ & 4 & 8 & 8 & 2 & 3 & 3 & 4 & 3 & 2 & 2 & 1,5 & 3 \\
\hline \multirow{2}{*}{ IV } & $\mathrm{s}$ & 4 & 0 & 0 & 5 & 4 & 4 & 3 & 7 & 6 & 5 & 5 & 5,5 \\
\hline & $\mathrm{i}$ & 3 & 10 & 9 & 2 & 3 & 3 & 4 & 2,5 & 2 & 3 & 1 & 1 \\
\hline
\end{tabular}




\section{Niveaux préférentiels}

En ce qui concerne les poissons nourris avec des Tubifex (Tableau 3), il existe une différence significative entre les moyennes des niveaux supérieurs et des niveaux inférieurs pour chacune des trois périodes $\mathrm{AS}, \mathrm{PS}_{1}$ et $\mathrm{PS}_{2}$ chez les animaux observés en groupe. Il en va de même chez les individus isolés, sauf pour la période AS. Le niveau préférentiel étant le niveau inférieur, la polarisation sur le substrat est confirmée lorsqu'on utilise une nourriture descendant par gravité. L'absence de niveau préféntiel observée chez les poissons isolés en période AS pourrait s'expliquer par une augmentation de l'exploration dans toutes les zones de l'aquarium lorsque l'individu ne repère pas la présence de congénères. Cette augmentation serait l'indice d'une anxiété accrue lorsque les effets d'apaisement par intégration au groupe sont impossibles.

\section{Tableau 3}

Comparaison des médians obtenues sur $A$. jordani pour les 3 niveaux au cours des 3 périodes. Poissons nourris antérieurement et stimulés avec des Tubifex.

\begin{tabular}{ccccc}
\hline \multicolumn{2}{c}{$\begin{array}{c}\text { Niveaux } \\
\text { comparés }\end{array}$} & & $\begin{array}{c}P \\
\text { (Poissons en groupe) }\end{array}$ & $\begin{array}{c}P \\
\text { (Poissons isolés) }\end{array}$ \\
\cline { 5 - 5 } $\mathrm{AS}_{\mathrm{S}}$ & $\mathrm{AS}_{\mathrm{i}}$ & & 0,029 & 0,171 \\
$\mathrm{PS}_{1 \mathrm{~s}}$ & $\mathrm{PS}_{1 \mathrm{i}}$ & 0,014 & 0,014 \\
$\mathrm{PS}_{2 \mathrm{~s}}$ & $\mathrm{PS}_{2 \mathrm{i}}$ & 0,014 & 0,014 \\
\hline
\end{tabular}

La même analyse, effectuée sur les poissons nourris avec de la nourriture flottante montre qu'il existe dans ce cas encore, une différence significative entre les moyennes des niveaux supérieurs et des niveaux inférieurs pour les 3 périodes considérées. Dans ces conditions toutefois, c'est le niveau supérieur qui est le niveau préférentiel, sauf en $\mathrm{PS}_{2}$, où les réactions sont orientées vers le niveau inférieur. Comme dans les expériences où l'on utilisait des Tubifex, il n'existe pas de niveau préférentiel en période AS chez les poissons isolés (Tableau 4).

La comparaison des résultats obtenus avec des Tubifex et avec de la nourriture flottante montre que l'orientation principale de l'exploration peut être modifiée par le niveau auquel se situe la nourriture après la phase chémoréceptrice initiale. Cependant, la réaction préférentielle étant à nouveau orientée vers le substrat en $\mathrm{PS}_{2}$, il semble que l'inversion observée ne soit qu'un phénomène temporaire, au moins chez les poissons observés en groupe. Le rétablissement de l'exploration vers le subtrat se manifeste, nous l'avons déjà dit, vers la 6ème minute, c'est-à-dire peu après le début de $\mathrm{PS}_{1}$.

Les réactions des Anoptichthys à un choc mécanique appliqué à la paroi de l'aquarium permettent d'établir certaines différences significatives entre niveaux, 


\section{Tableau 4}

Comparaison des médians obtenus sur $A$. jordani pour les 3 niveaux au cours des 3 périodes. Poissons nourris antérieurement et stimulés avec de la nourriture flottante.

\begin{tabular}{|c|c|c|c|}
\hline \multicolumn{2}{|c|}{$\begin{array}{l}\text { Niveaux } \\
\text { comparés }\end{array}$} & \multirow{2}{*}{$\begin{array}{c}\mathrm{P} \\
\frac{\text { (Poissons en groupe) }}{0,014}\end{array}$} & \multirow{2}{*}{$\begin{array}{c}\mathrm{P} \\
\text { (Poissons isolés) } \\
0,343\end{array}$} \\
\hline $\mathrm{AS}_{\mathrm{S}}$ & $\mathrm{AS}_{\mathrm{i}}$ & & \\
\hline $\mathrm{PS}_{1 \mathrm{~s}}$ & $\mathrm{PS}_{1 \mathrm{i}}$ & 0,014 & 0,014 \\
\hline $\mathrm{PS}_{2 \mathrm{~s}}$ & $\mathrm{PS}_{2 \mathrm{i}}$ & 0,014 & 0,014 \\
\hline
\end{tabular}

tant chez les poissons nourris antérieurement avec des Tubifex, que chez ceux qui avaient été nourris avec des Daphnies (Tableau 5). Chez les premiers, il existe une différence significative en faveur du niveau inférieur pour les 3 périodes dans le cas des animaux observés en groupe. Chez les individus isolés, la différence n'est significative qu'en $\mathrm{PS}_{1}$; elle montre une réaction préférentielle pour le niveau supérieur.

\section{Tableau 5}

Comparaison des médians obtenus sur $A$. jordani pour les 3 niveaux au cours des 3 périodes.

A - Poissons nourris avec des Tubifex et stimulés par un choc mécanique

\begin{tabular}{|c|c|c|c|}
\hline \multicolumn{2}{|c|}{$\begin{array}{l}\text { Niveaux } \\
\text { comparés }\end{array}$} & $\begin{array}{c}\mathrm{P} \\
\text { (Poissons en groupe) }\end{array}$ & $\begin{array}{c}\mathrm{P} \\
\text { (Poissons isolés) }\end{array}$ \\
\hline $\mathrm{AS}_{\mathrm{S}}$ & $\mathrm{AS}_{\mathrm{i}}$ & 0,014 & 0,057 \\
\hline $\mathrm{PS}_{1 \mathrm{~s}}$ & $\mathrm{PS}_{1 \mathrm{i}}$ & 0,014 & 0,014 \\
\hline $\mathrm{PS}_{2 \mathrm{~s}}$ & $\mathrm{PS}_{2 \mathrm{i}}$ & 0,014 & 0,171 \\
\hline
\end{tabular}

B - Poissons nourris avec de la nourriture flottante et stimulés par un choc mécanique

\begin{tabular}{llll}
$\mathrm{AS}_{\mathrm{S}}$ & $\mathrm{AS}_{\mathrm{i}}$ & 0,029 & 0,014 \\
$\mathrm{PS}_{1 \mathrm{~s}}$ & $\mathrm{PS}_{1 \mathrm{i}}$ & 0,014 & 0,014 \\
$\mathrm{PS}_{2 \mathrm{~s}}$ & $\mathrm{PS}_{2 \mathrm{i}}$ & 0,014 & 0,014 \\
\hline
\end{tabular}


Chez les seconds, toutes les différences sont significatives et manifestent une réaction préférentielle pour le niveau supérieur, tant chez les poissons en groupe que chez les poissons isolés.

\section{Evolution des réactions au cours des 3 périodes d'observation $A S, P S_{1}, P S_{2}$}

L'analyse statistique des résultats obtenus au cours des trois périodes d'observation permet d'évaluer les modifications significatives survenues dans les comportements avant la stimulation et après celle-ci dans les diverses conditions d'observation.

Chez les individus nourris antérieurement avec des Tubifex et stimulés avec cette même nourriture, le passage de $\mathrm{AS}$ à $\mathrm{PS}_{1}$ se traduit par une modification significative de la densité des animaux observés en groupe, tant au niveau supérieur qu'au niveau inférieur. Comme le montrent les graphiques, cette modification entraîne une augmentation de densité au niveau inférieur au cours de $\mathrm{PS}_{1}$.

De $\mathrm{PS}_{1}$ à $\mathrm{PS}_{2}$, l'évolution est significative uniquement au niveau inférieur, dans le sens d'une réduction de densité en $\mathrm{PS}_{2}$. De $\mathrm{AS}_{\text {à }} \mathrm{PS}_{2}$, l'évolution est significative pour les deux niveaux considérés, dans le sens d'une réduction de densité au niveau supérieur, avec accroissement corrélatif de densité au niveau inférieur.

Chez les indivius isolés, l'évolution est la même de $\mathrm{AS}$ à $\mathrm{PS}_{1}$ et de $\mathrm{AS}$ à $\mathrm{PS}_{2}$. Par contre, aucune différence significative n'apparaît de $\mathrm{PS}_{1}$ à $\mathrm{PS}_{2}$ (Tableau 6).

\section{Tableau 6}

Evolution des réactions d'A. jordani au cours de $\mathrm{AS}, \mathrm{PS}_{1}$ et $\mathrm{PS}_{2}$. Poissons nourris antérieurement et stimulés avec des Tubifex.

\begin{tabular}{llcc}
\hline \multicolumn{2}{c}{$\begin{array}{c}\text { Périodes } \\
\text { comparées }\end{array}$} & $\begin{array}{c}\mathrm{P} \\
\text { (Poissons en groupe) }\end{array}$ & $\begin{array}{c}\mathrm{P} \\
\text { (Poissons isolés) }\end{array}$ \\
\cline { 2 - 3 } $\mathrm{AS}_{\mathrm{S}}$ & $\mathrm{PS}_{1 \mathrm{~s}}$ & 0,014 & 0,014 \\
$\mathrm{AS}_{\mathrm{i}}$ & $\mathrm{PS}_{1 \mathrm{i}}$ & 0,014 & 0,014 \\
$\mathrm{PS}_{1 \mathrm{~s}}$ & $\mathrm{PS}_{2 \mathrm{~s}}$ & Pas de différence & 0,057 \\
$\mathrm{PS}_{1 \mathrm{i}}$ & $\mathrm{PS}_{2 \mathrm{i}}$ & 0,029 & Pas de différence \\
$\mathrm{AS}_{\mathrm{S}}$ & $\mathrm{PS}_{2 \mathrm{~s}}$ & 0,014 & 0,014 \\
$\mathrm{AS}_{\mathrm{i}}$ & $\mathrm{PS}_{2 \mathrm{i}}$ & 0,014 & 0,014 \\
\hline
\end{tabular}

Chez les individus nourris antérieurement avec de la nourriture flottante et stimulés avec celle-ci, on constate également des modifications marquées d'une période à l'autre. Les onnées statistiques relatives à ces conditions figurent au tableau 7. Dans le cas des animaux observés en groupe, on constate, ici encore, une modification de densité significative de $\mathrm{AS}$ à $\mathrm{PS}_{1}$, tant pour le niveau inférieur que pour le niveau supérieur. La même constation s'impose de $\mathrm{PS}_{1}$ à $\mathrm{PS}_{2}$ et de $\mathrm{AS}$ et $\mathrm{PS}_{2}$. 
Chez les individus isolés, l'évolution de la densité est sigficative de $\mathrm{AS}$ à $\mathrm{PS}_{1}$ pour les deux considérés. De $\mathrm{PS}_{1}$ à $\mathrm{PS}_{2}$ et de $\mathrm{AS}$ à $\mathrm{PS}_{2}$ la densité s'accroît de façon significative au niveau supérieur seulement.

\section{Tableau 7}

Evolution des réactions d' $A$. jordani au cours de $\mathrm{AS}, \mathrm{PS}_{1}$ et $\mathrm{PS}_{2}$. Poissons nourris antérieurement et stimulés avec de la nourriture flottante.

\begin{tabular}{llcc}
\hline \multicolumn{2}{c}{$\begin{array}{c}\text { Périodes } \\
\text { comparées }\end{array}$} & $\begin{array}{c}\mathrm{P} \\
\text { (Poissons en groupe) }\end{array}$ & $\begin{array}{c}\mathrm{P} \\
\text { (Poissons isolés) }\end{array}$ \\
$\mathrm{AS}_{\mathrm{S}}$ & $\mathrm{PS}_{1 \mathrm{~s}}$ & 0,014 & 0,014 \\
$\mathrm{AS}_{\mathrm{i}}$ & $\mathrm{PS}_{1 \mathrm{i}}$ & 0,014 & 0,014 \\
$\mathrm{PS}_{1 \mathrm{~s}}$ & $\mathrm{PS}_{2 \mathrm{~s}}$ & 0,014 & 0,029 \\
$\mathrm{PS}_{1 \mathrm{i}}$ & $\mathrm{PS}_{2 \mathrm{i}}$ & 0,014 & 0,171 \\
$\mathrm{AS}_{\mathrm{S}}$ & $\mathrm{PS}_{2 \mathrm{~s}}$ & 0,014 & 0,014 \\
$\mathrm{AS}_{\mathrm{i}}$ & $\mathrm{PS}_{2 \mathrm{i}}$ & 0,014 & 0,057 \\
\hline
\end{tabular}

Le phénomène principal mis en évidence est donc un accroissement significatif de densité au niveau supérieur. Les poissons isolés se maintiennent à ce niveau au cours de $\mathrm{PS}_{2}$, tandis que les poissons en groupe tendent à le quiter, tout en y conservant une densité plus forte qu'en AS.

Envisageons à présent l'évolution des réactions d'une période à l'autre lorsque l'on utilise un stimulus mécanique. Les résultats statistiques, groupés aux tableaux 8

\section{Tableau 8}

Evolution des réactions d'A. jordani au cours de $\mathrm{AS}, \mathrm{PS}_{1}$ et $\mathrm{PS}_{2}$. Poissons nourris antérieurement avec des Tubifex et stimulés par un choc mécanique.

\begin{tabular}{|c|c|c|c|c|}
\hline \multicolumn{2}{|c|}{$\begin{array}{l}\text { Périodes } \\
\text { comparées }\end{array}$} & $\begin{array}{c}\text { P } \\
\text { (Poissons en groupe) }\end{array}$ & $\begin{array}{c}\text { P } \\
\text { (Poissons isolés) }\end{array}$ & \\
\hline $\mathrm{AS}_{\mathrm{S}}$ & $\mathrm{PS}_{1 \mathrm{~s}}$ & 0,100 & 0,029 & \\
\hline $\mathrm{AS}_{\mathrm{i}}$ & $\mathrm{PS}_{1 \mathrm{i}}$ & 0,029 & 0,557 & , \\
\hline $\mathrm{PS}_{1 \mathrm{~s}}$ & $\mathrm{PS}_{2 \mathrm{~s}}$ & 0,100 & 0,243 & \\
\hline $\mathrm{PS}_{1 \mathrm{i}}$ & $\mathrm{PS}_{2 \mathrm{i}}$ & 0,029 & 0,171 & \\
\hline $\mathrm{AS}_{\mathrm{S}}$ & $\mathrm{PS}_{2 \mathrm{~s}}$ & 0,100 & 0,171 & \\
\hline $\mathrm{AS}_{\mathrm{i}}$ & $\mathrm{PS}_{2 \mathrm{i}}$ & 0,100 & 0,443 & \\
\hline
\end{tabular}


et 9 , fournissent des indications très différentes de celles que l'on obtient lorsque l'on utilise des stimuli chimiques.

Chez les poissons nourris antérieurement avec des Tubifex, les deux seules différences significatives relevées dans les groupes concernent la réduction de densité au niveau inférieur de $\mathrm{AS}$ à $\mathrm{PS}_{1}$ et l'accroissement de densité à ce même niveau de $\mathrm{PS}_{1}$ à $\mathrm{PS}_{2}$. On ne note aucune différence marquante dans la densité de $\mathrm{AS}$ à $\mathrm{PS}_{2}$. Chez les poissons isolés, l'intervention du choc mécanique se traduit par une réduction significative de la présence au niveau supérieur au cours de $\mathrm{PS}_{1}$. Pour le reste, les réactions des poissons isolés au choc mécanique ne présentent aucune évolution typique de $\mathrm{AS}$ à $\mathrm{PS}_{2}$.

En ce qui concerne les poissons nourris antérieurement avec de la nourriture flottante, les différences significatives sont également peu fréquentes. Chez les animaux observés en groupe, les deux seules valeurs significatives trouvées indiquent une décroissance de densité au niveau inférieur de $\mathrm{PS}_{1}$ à $\mathrm{PS}_{2}$ et de $\mathrm{AS}$ à $\mathrm{PS}_{2}$. Chez les poissons isolés, l'évolution est nette de $\mathrm{AS}$ à $\mathrm{PS}_{1}$. Elle indique une présence plus fréquente en $\mathrm{PS}_{1}$ au niveau supérieur et moins fréquente au niveau inférieur.

\section{Tableau 9}

Evolution des réactions d' $A$. jordani au cours de AS, $\mathrm{PS}_{1}$ et $\mathrm{PS}_{2}$. Poissons nourris antérieurement avec de la nourriture flottante et stimulés par un choc mécanique.

\begin{tabular}{|c|c|c|c|}
\hline \multicolumn{2}{|c|}{$\begin{array}{l}\text { Périodes } \\
\text { comparées }\end{array}$} & \multirow{2}{*}{$\begin{array}{c}\mathrm{P} \\
\frac{\text { (Poissons en groupe) }}{0,171}\end{array}$} & \multirow{2}{*}{$\begin{array}{c}\mathrm{P} \\
\text { (Poissons isolés) } \\
0,029\end{array}$} \\
\hline $\mathrm{AS}_{\mathrm{S}}$ & $\mathrm{PS}_{1 \mathrm{~s}}$ & & \\
\hline $\mathrm{AS}_{\mathrm{i}}^{\mathrm{s}}$ & $\mathrm{PS}_{1 \mathrm{i}}$ & 0,171 & 0,014 \\
\hline $\mathrm{PS}_{1 \mathrm{~s}}$ & $\mathrm{PS}_{2 \mathrm{~s}}$ & 0,057 & 0,100 \\
\hline $\mathrm{PS}_{1 \mathrm{i}}$ & $\mathrm{PS}_{2 \mathrm{i}}$ & 0,014 & 0,171 \\
\hline $\mathrm{AS}_{\mathrm{S}}$ & $\mathrm{PS}_{2 \mathrm{~s}}$ & 0,171 & 0,100 \\
\hline $\mathrm{AS}_{\mathrm{i}}$ & $\mathrm{PS}_{2 \mathrm{i}}$ & 0,014 & 0,171 \\
\hline
\end{tabular}

Le fait essentiel qui se dégage des expériences utilisant un choc mécanique comme stimulus est une différenciation moins marquée des comportements en ce qui concerne l'évolution d'une période d'observation à la suivante. Quant au niveau préférentiel, il est étroitement lié au type de nourriture. En effet, malgré l'absence généralisée d'exploration active, les poissons stimulés par un choc mécanique ont tendance à se porter plus souvent au niveau inférieur lorsqu'ils ont été nourris antérieurement avec des Tubifex, et au niveau supérieur lorsqu'ils ont été nourris antérieurement avec de la nourriture flottante.

La comparaison statistique des deux catégories de poissons (nourris antérieurement avec des Tubifex et nourris antérieurement avec de la nourriture flottante) confirme entièrement les données trouvées sur Anoptichthys dans les différentes conditions expérimentales. 


\section{II - RESULTATS OBTENUS SUR CAECOBARBUS GEERTSI}

\section{A. Description des comportements}

\section{Période antérieure à l'intervention du stimulus (AS)}

Les observations pratiquées au cours de cette période mettent en évidence des comportements légèrement différents de ceux relevés chez Anoptichthys. Les Caecobarbus répartissent, eux aussi, leur activé de façon assez irrégulière aux troix niveaux et manifestent également une préférence pour les niveaux moyen et inférieur. Toutefois, contrairement aux Anoptichthys, les Caecobarbus ont tendance à se répartir préférentiellement à ces niveaux, même s'ils ont été nourris antérieurement avec de la nourriture flottante. Ajoutons que la nage

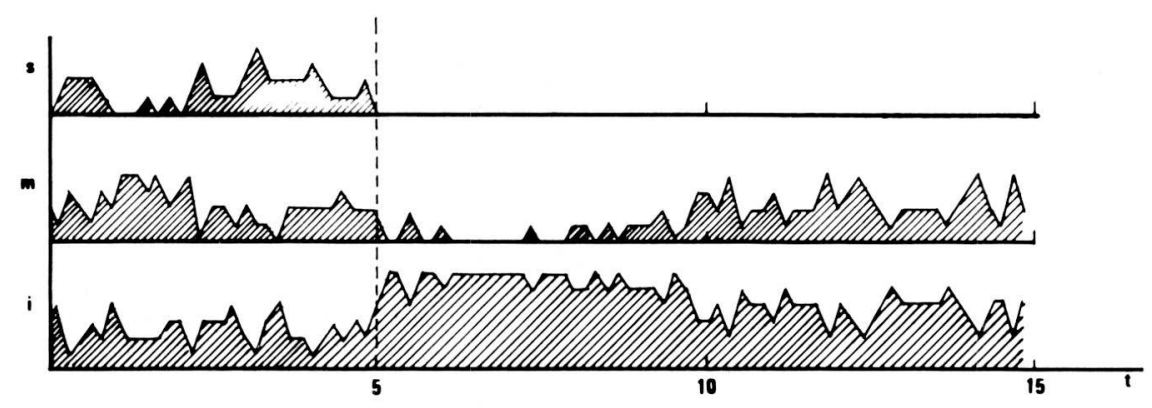

Fig. 6a. Distribution verticale caractéristique d'un groupe de 6 Caecobarbus nourris antérieurement et stimulés avec des Tubifex. Abscisses: temps en minutes. Ordonnées: nombre de poissons (f) en valeur absolue. i,m,s: niveaux respectifs inférieur, moyen et supérieur. Le trait vertical en pointillé indique le moment d'intervention du stimulus.

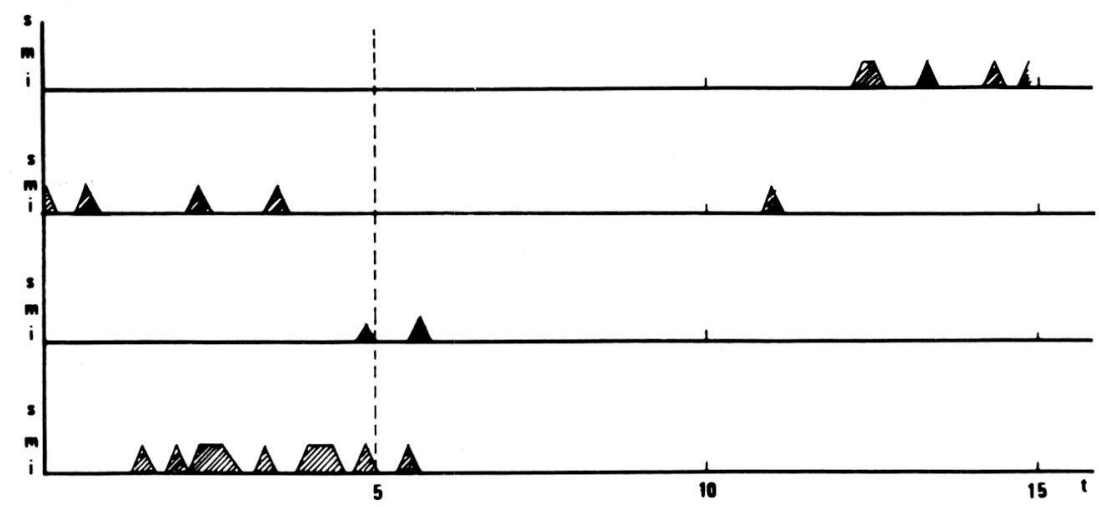

Fig. 6b. Distribution caractéristique des positions verticales d'un Caecobarbus observé individuellement (nourri antérieurement et stimulé avec des Tubifex). Relevé de 4 expériences identiques. Mêmes indications qu'en (a). 
des Caecobarbus est plus lente et que l'activité générale est moindre que chez Anoptichthys.

\section{Période postérieure à l'intervention du stimulus $\left(P S_{1}+P S_{2}\right)$}

Tant chez les individus isolés que dans les groupes, l'intervention de la stimulation chimique provoque une accélération de la nage, qu'il s'agisse de poissons nourris antérieurement avec des Tubifex ou de poissons nourris antérieurement avec de la nourriture flottante. Les premiers plongent ensuite brusquement vers le fond à proximité du point d'impact de la nourriture, mais l'efficacité de l'exploration est très variable. Il arrive souvent que l'animal cotoie la nourriture sans la saisir et même sans manifester de recherche active. Quant aux seconds, au lieu de plonger

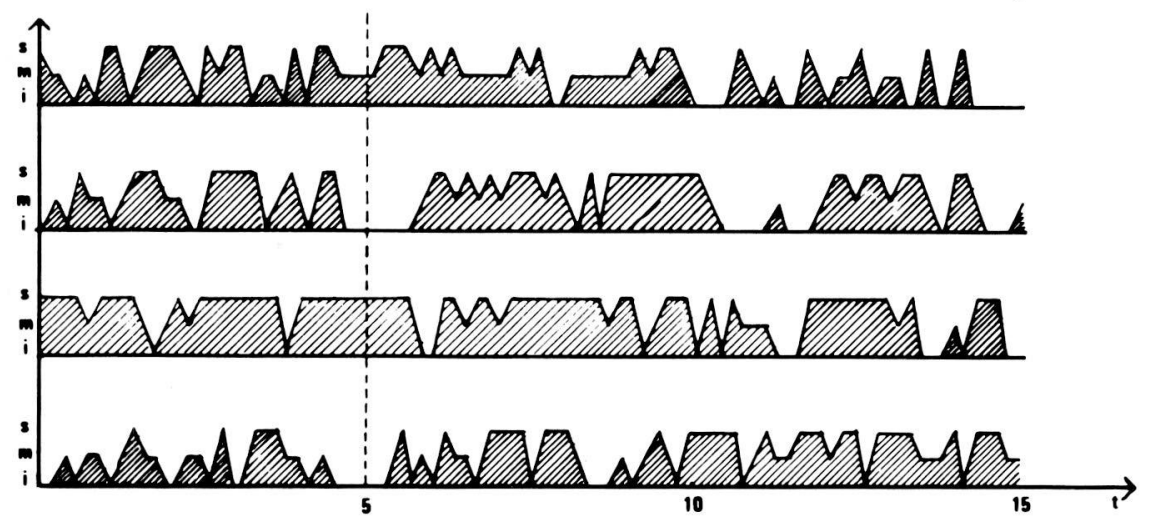

Fig. 7a. Distribution verticale caractéristique d'un groupe de 6 Caecobarbus nourris antérieurement et stimulés avec de la nourriture flottante. Abscisses: temps en minutes. Ordonnées: nombre de poissons (f) en valeur absolue. i,m,s: niveaux respectifs inférieur, moyen et supérieur. Le trait vertical en pointillé indique le moment d'intervention du stimulus.

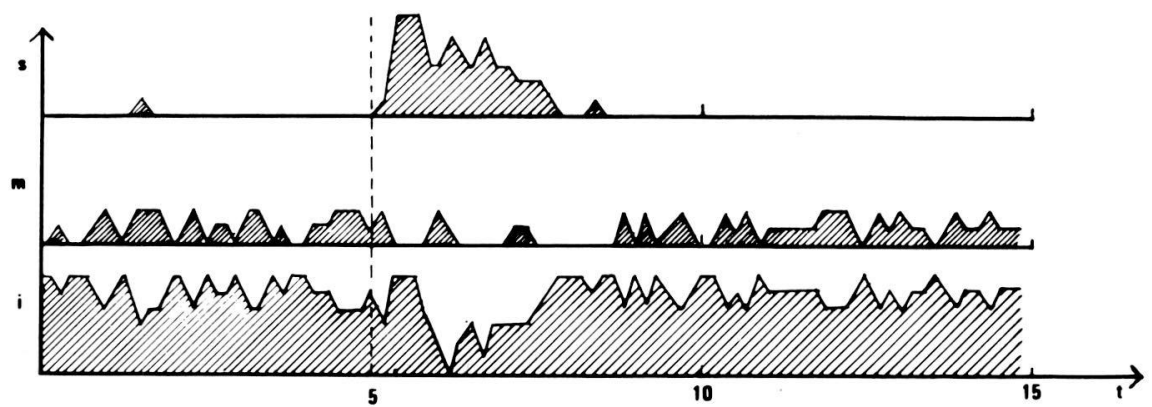

Fig. 7b. Distribution caractéristique des positions verticales d'un Caecobarbus observé individuellement (nourri antérieurement et stimulé de la nourriture flottante). Relevé de 4 expériences identiques. Mêmes indications qu'en (a). 


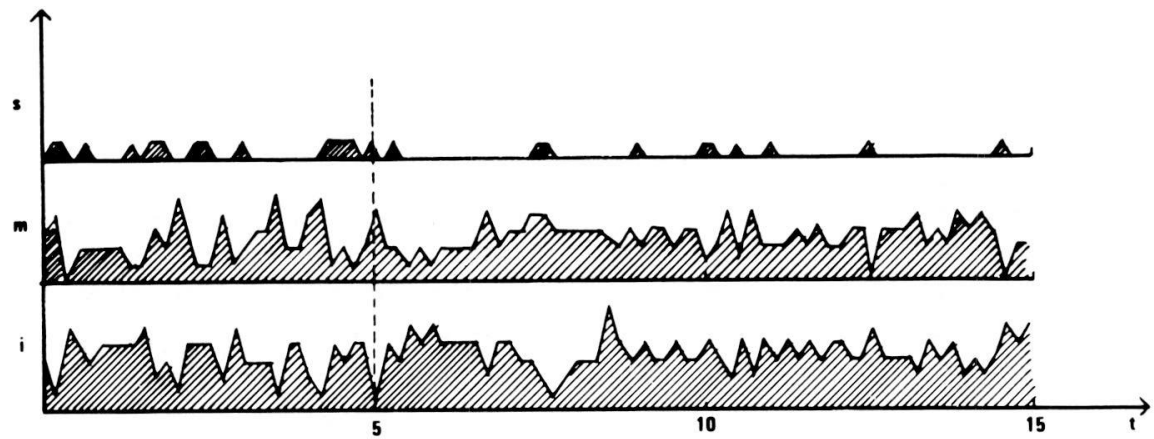

Fig. 8a. Distribution verticale caractéristique d'un groupe de 6 Caecobarbus nourris antérieurement avec des Tubifex et stimulés par un choc mécanique. Abscisses: temps en minutes. Ordonnées: nombre de poissons (f) en valeur absolue. i,m,s: niveaux respectifs inférieur, moyen et supérieur. Le trait vertical en pointillé indique le moment d'intervention du stimulus.

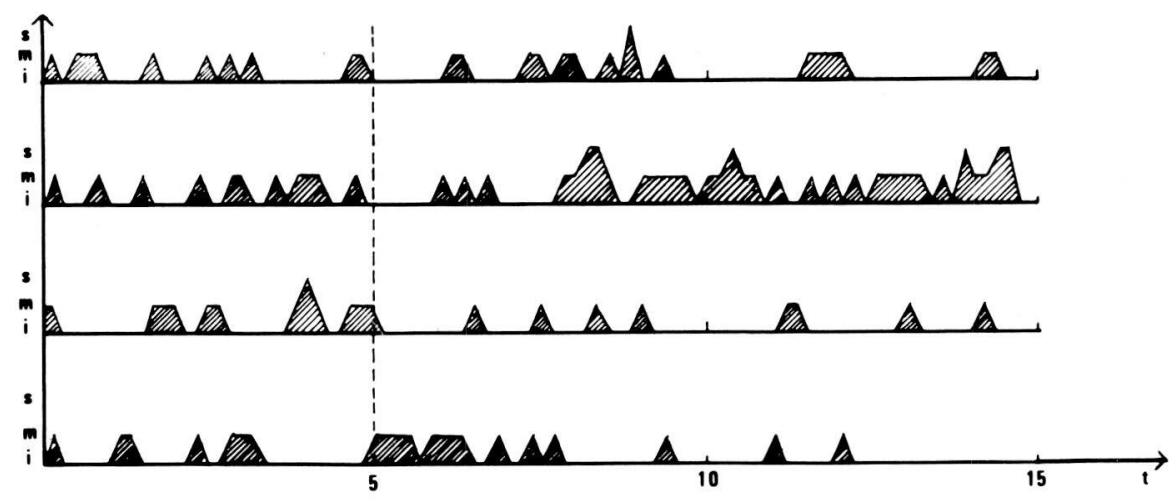

Fig. 8b. Distribution caractéristique des positions verticales d'un Caecobarbus observé individuellement (nourri antérieurement avec des Tubifex et stimulé par un choc mécanique). Relevé de 4 expériences identiques. Mêmes indications qu'en (a).

vers le substrat, ils gagnent la surface en décrivant de grands cercles et en exécutant des mouvements fréquents de happement au cours de la nage.

$\mathrm{Au}$ cours de $\mathrm{PS}_{2}$, les poissons des deux catégories procèdent à une exploration sur le fond. Chez les individus isolés nourris antérieurement avec de la nourriture flottante, on observe de brusques remontes vers la surface.

Chez les Caecobarbus nourris antérieurement et stimulés avec des Tubifex, une tendance au rétablissement de la répartition observée an AS apparaît vers la 9ème ou la 10ême minute (fin de la période $\mathrm{PS}_{1}$ ).

Comme chez les Anoptichthys, la stimulation mécanique provoque chez les Caecobarbus une accélération de la nage dans toutes les directions (comportement phobique), accompagnée d'une tendance à gagner préférentiellement le niveau 
inférieur dès le début de $\mathrm{PS}_{1}$, celle-ci étant plus marquée chez les poissons observés en groupe. Les résultats sont semblables pour les poissons nourris antérieurement avec des Tubifex et pour ceux nourris antérieurement avec de la nourriture flottante. Aucun indice d'exploration systématique n'apparaît dans ces conditions.

L'observation des comportements des Caecobarbus révèle donc les faits essentiels suivants:

1. Comme chez les Anoptichthys, les stimuli chimiques et le stimulus mécanique provoquent tous une accélération de la nage.

2. Comme chez les Anoptichthys, seuls les stimuli chimiques provoquent une accélération de la nage suivie d'exploration. Chez les Caecobarbus toutefois, l'exploration est moins active, moins précise et plus irrégulière.

3. Le stimulus mécanique provoque chez les Caecobarbus les mêmes effets que chez les Anoptichthys: accélération de la nage suivie de fuite.

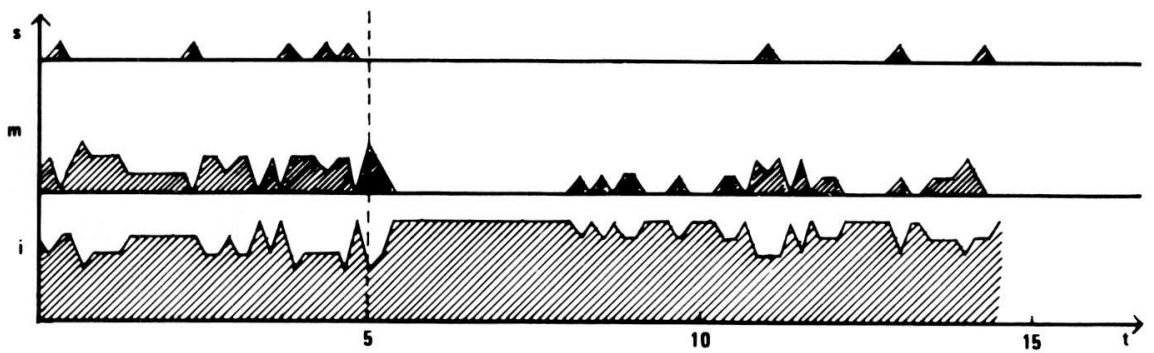

Fig. 9. Distribution verticale caractéristique d'un groupe de 6 Caecobarbus nourris antérieurement avec de la nourriture flottante et stimulés par un choc mécanique. Abscisses: temps en minutes. Ordonnées: nombre de poissons (f) en valeur absolue. i,m,s: niveaux respectifs inférieur, moyen et supérieur. Le trait vertical en pointillé indique le moment d'intervention du stimulus.

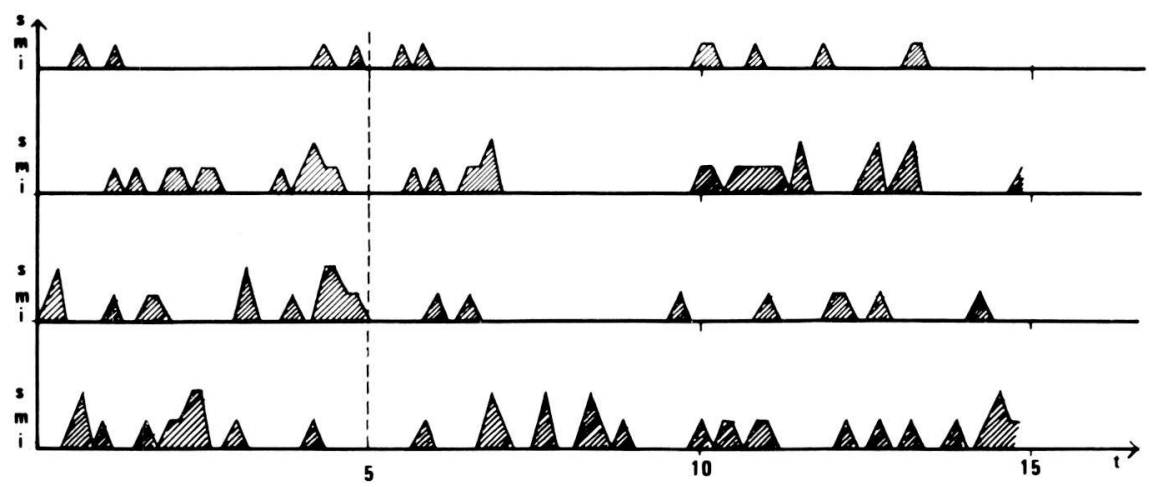

Fig. 9b. Distribution caractéristique des positions verticales d'un Caecobarbus observé individuellement (nourri antérieurement avec de la nourriture flottante et stimulé par un choc mécanique). Relevé de 4 expériences identiques. Mêmes indications qu'en (a). 
4. Contrairement à ce qui a été observé chez les Anoptichthys, le niveau préférentiel d'exploration n'est pas déterminé par le type nourriture utilisé comme stimulus. Les poissons nourris antérieurement avec des Tubifex et ceux nourris antérieurement avec de la nourriture flottante ont tendance, les uns et les autres à gagner préférentiellement le niveau inférieur, bien que l'exploration du substrat soit moins efficace que chez Anoptichthys.

\section{B. Analyse statistique des résultats.}

Les données d'observations obtenues sur Caecobarbus ont été soumises aux mêmes traitements statistiques que ceux qui ont été appliqués à propos d'Anoptichthys. Les moyennes sont données au tableau 10 pour les Caecobarbus observés en groupe et au tableau 11 pour les Caecobarbus isolés.

\section{Tableau 10}

Moyennes des observations effectuées sur Caecobarbus geertsi dans les diverses conditions expérimentales. Poissons observés en groupe.

\begin{tabular}{|c|c|c|c|c|c|c|c|c|c|c|c|c|c|}
\hline \multirow{2}{*}{ séance } & \multirow{2}{*}{$\begin{array}{l}\text { Sti. } \\
\overline{\text { Pér. }} \\
\text { Niv. }\end{array}$} & \multicolumn{3}{|c|}{$\begin{array}{l}\text { Nourris \& Sti. } \\
\text { avec Tubifex }\end{array}$} & \multicolumn{3}{|c|}{$\begin{array}{l}\text { Nourri. Tub. } \\
\text { stim. Choc } \\
\end{array}$} & \multicolumn{3}{|c|}{$\begin{array}{l}\text { Nourri. flott. } \\
\text { stim. flott. }\end{array}$} & \multicolumn{3}{|c|}{$\begin{array}{l}\text { Nourri. flott. } \\
\text { stim. Choc }\end{array}$} \\
\hline & & AS & $\mathrm{PS}_{1}$ & $\mathrm{PS}_{2}$ & AS & $\mathrm{PS}_{1}$ & $\mathrm{PS}_{2}$ & AS & $\mathrm{PS}_{1}$ & $\mathrm{PS}_{2}$ & AS & $\mathrm{PS}_{1}$ & $\mathrm{PS}_{2}$ \\
\hline \multirow{2}{*}{ I } & s & 0 & 0 & 0 & 0 & 0 & 0 & 3 & 3 & 2 & 1,5 & 2 & 3 \\
\hline & $\mathrm{i}$ & 4 & 5 & 5 & 4 & 3 & 3 & 2 & 3 & 2 & 3 & 3 & 1 \\
\hline \multirow{2}{*}{ II } & $\mathrm{s}$ & 1 & 0 & 0 & 1 & 0 & 0 & 0 & 3 & 0 & 1 & 0 & 1 \\
\hline & $\mathrm{i}$ & 2 & 6 & 4 & 2 & 6 & 4 & 5 & 2 & 6 & 3 & 5 & 3 \\
\hline \multirow{2}{*}{ III } & s & 0 & 0 & 0 & 0 & 0 & 0 & 0 & 1 & 0 & 0 & 0 & 0 \\
\hline & $\mathrm{i}$ & 3 & 6 & 5 & 3 & 6 & 5 & 5 & 4 & 5 & 4,5 & 6 & 5,5 \\
\hline \multirow{2}{*}{ IV } & s & 0 & 0 & 0 & 0 & 0 & 0 & 0 & 3 & 0 & 0 & 0 & 0 \\
\hline & $\mathrm{i}$ & 6 & 6 & 6 & 6 & 6 & 6 & 4 & 3 & 5 & 6 & 5 & 6 \\
\hline
\end{tabular}




\section{Tableau 11}

Moyennes des observations effectuées sur Caecobarbus geertsi dans les diverses conditions expérimentales. Poissons observés individuellement.

\begin{tabular}{|c|c|c|c|c|c|c|c|c|c|c|c|c|c|}
\hline \multirow[t]{2}{*}{ séance } & \multirow{2}{*}{$\begin{array}{l}\text { Sti. } \\
- \\
\text { Pér. } \\
\text { Niv. }\end{array}$} & \multicolumn{3}{|c|}{$\begin{array}{l}\text { Nourris \& Sti. } \\
\text { avec Tubifex }\end{array}$} & \multicolumn{3}{|c|}{$\begin{array}{l}\text { Nourri. Tub. } \\
\text { stim. Choc }\end{array}$} & \multicolumn{3}{|c|}{$\begin{array}{l}\text { Nourri. Flott. } \\
\text { stim. flott. }\end{array}$} & \multicolumn{3}{|c|}{$\begin{array}{l}\text { Nourri. Flott. } \\
\text { stim. Choc }\end{array}$} \\
\hline & & AS & $\mathrm{PS}_{1}$ & $\mathrm{PS}_{2}$ & $\mathrm{~A}_{\mathrm{S}}$ & $\mathrm{PS}_{1}$ & $\mathrm{PS}_{2}$ & AS & $\mathrm{PS}_{1}$ & $\mathrm{PS}_{2}$ & AS & $\mathrm{PS}_{1}$ & $\mathrm{PS}_{2}$ \\
\hline \multirow{2}{*}{ I } & s & 0 & 0 & 0 & 0 & 0 & 0 & 0,5 & 2 & 0 & 1 & 0 & 0 \\
\hline & $\mathrm{i}$ & 3 & 6 & 5 & 3 & 4 & 3,5 & 4 & 2,5 & 4 & 3 & 4 & 3 \\
\hline \multirow{2}{*}{ II } & $\mathrm{s}$ & 0 & 0 & 0 & 0 & 0 & 0 & 1 & 2 & 3 & 0 & 0 & 0 \\
\hline & $\mathrm{i}$ & 5 & 6 & 6 & 3 & 4 & 4 & 4 & 2 & 3 & 3 & 5 & 4 \\
\hline \multirow{2}{*}{ III } & $\mathrm{s}$ & 0 & 0 & 0 & 1 & 0 & 0 & 0 & 2 & 1 & 1 & 0 & 0 \\
\hline & $\mathrm{i}$ & 5 & 6 & 6 & 3 & 3,5 & 3 & 4 & 2 & 3 & 4 & 5 & 4 \\
\hline \multirow{2}{*}{ IV } & $\mathrm{s}$ & 0 & 0 & 0 & 0 & 0 & 0 & 0 & 2 & 0 & 1 & 0 & 0,5 \\
\hline & $\mathrm{i}$ & 5 & 6 & 6 & 4 & 4 & 4 & 5 & 2 & 4 & 3 & 4,5 & 4 \\
\hline
\end{tabular}

\section{Niveaux préférentiels}

Les poissons nourris antérieurement avec des Tubifex et stimulés avec ceux-ci, manifestent une préférence significative pour le niveau inférieur $(P=0,014)$ dans chacune des 3 périodes $\mathrm{AS}, \mathrm{PS}_{1}$ et $\mathrm{PS}_{2}$. Ceci s'observe tant chez les animaux en groupe que chez les animaux isolés. La différence essentielle par rapport aux Anoptichthys concerne donc la période AS, au cours de laquelle ces derniers, isolés, ne manifestent pas de présence préférentielle au niveau inférieur.

L'analyse des données obtenues sur les Caecobarbus nourris antérieurement et stimulés avec de la nourriture flottante, fournit des indications indentiques en ce qui concerns $\mathrm{AS}$ et $\mathrm{PS}_{2}$ : les poissons, tant en groupe qu'isolés, se portent significativement vers le substrat. Par contre, aucun des deux niveaux extrêmes n'est visité préférentiellement en $\mathrm{PS}_{1}$ (Tableau 12).

L'absence de répartition verticale caractéristique au cours de $\mathrm{PS}_{1}$ est due au fait qu'immédiatement après la stimulation par nourriture flottante, les Caecobarbus nagent en cercle en passant du substrat à la surface et inversement pendant une minute environ, sans s'établir à un niveau défini.

Quant aux réactions des Caecobarbus à la stimulation mécanique, elles sont beaucoup plus homogènes que celles des Anoptichthys. Les valeurs statistiques indiquent dans toutes les conditions d'observation que la préférence des Caecobarbus pour le niveau inférieur, observée en AS, se maintient au cours de $\mathrm{PS}_{1}$ et de $\mathrm{PS}_{2}(\mathrm{P}=0,014$ dans 11 comparaisons sur 12 , la valeur restante étant de $\mathrm{P}=0,029)$. 


\section{Tableau 12}

Comparaison des médians obtenus sur $C$. geertsi pour les 3 niveaux au cours des 3 périodes. Poissons nourris antérieurement et stimulés avec de la nourriture flottante.

\begin{tabular}{|c|c|c|c|}
\hline \multicolumn{2}{|c|}{$\begin{array}{l}\text { Niveaux } \\
\text { comparés }\end{array}$} & $\begin{array}{c}\mathrm{P} \\
\text { (Poissons en groupe) }\end{array}$ & $\begin{array}{c}\mathrm{P} \\
\text { (Poissons isolés) }\end{array}$ \\
\hline $\mathrm{AS}_{\mathrm{S}}$ & $\mathrm{AS}_{\mathrm{i}}$ & 0,029 & 0,014 \\
\hline $\mathrm{PS}_{1 \mathrm{~s}}$ & $\mathrm{PS}_{1 \mathrm{i}}$ & 0,100 & Pas de differénce \\
\hline $\mathrm{PS}_{2 \mathrm{~s}}$ & $\mathrm{PS}_{2 \mathrm{i}}$ & 0,014 & 0,014 \\
\hline
\end{tabular}

2. Evolution des réactions au cours des 3 périodes d'observation $A S, P S_{1}, P S_{2}$.

Le passage de AS à PS, entraîne chez les Caecobarbus nourris avec des Tubifex et stimulés avec cette même nourriture, une modification significative de la densité des animaux observés en groupe, tant au niveau supérieur qu'au niveau inférieur. L'évolution est la même que chez les Anoptichthys: elle se traduit par une augmentation de densité au niveau inférieur au cours de $\mathrm{PS}_{1}$. De $\mathrm{PS}_{1}$ à $\mathrm{PS}_{2}$, aucune évolution significative n'apparaît. De $\mathrm{AS}$ à $\mathrm{PS}_{2}$, il y a une réduction significative de la densité au niveau supérieur, mais aucun changement marquant en ce qui concerne le niveau inférieur.

\section{Tableau 13}

Evolution des réactions de $C$. geertsi au cous de $\mathrm{AS}, \mathrm{PS}_{1}$ et $\mathrm{PS}_{2}$. Poissons nourris antérieurement et stimulés avec des Tubifex.

\begin{tabular}{|c|c|c|c|}
\hline \multicolumn{2}{|c|}{$\begin{array}{l}\text { Périodes } \\
\text { comparées }\end{array}$} & $\begin{array}{c}\text { P } \\
\text { (Poissons en groupe) }\end{array}$ & $\begin{array}{c}\mathrm{P} \\
\text { (Poissons isolés) }\end{array}$ \\
\hline $\mathrm{AS}_{\mathrm{S}}$ & $\mathrm{PS}_{1 \mathrm{~s}}$ & 0,014 & Pas de différence \\
\hline $\mathrm{AS}_{\mathrm{i}}$ & $\mathrm{PS}_{1 \mathrm{i}}$ & 0,029 & 0,014 \\
\hline $\mathrm{PS}_{1 \mathrm{~s}}$ & $\mathrm{PS}_{2 \mathrm{~s}}$ & Pas de différence & Pas de différence \\
\hline $\mathrm{PS}_{1 \mathrm{i}}$ & $\mathrm{PS}_{2 \mathrm{i}}$ & 0,057 & 0,014 \\
\hline $\mathrm{AS}_{\mathrm{S}}$ & $\mathrm{PS}_{2 \mathrm{~s}}$ & 0,014 & Pas de différence \\
\hline $\mathrm{AS}_{\mathrm{i}}$ & $\mathrm{PS}_{2 \mathrm{i}}$ & 0,100 & 0,014 \\
\hline
\end{tabular}


L'analyse indique par conséquent un processus comparable à celui que l'on relève chez les Anoptichthys, avec cette différence que la densité au niveau inférieur atteinte en $\mathrm{PS}_{1}$ est plus stable chez les Caecobarbus et se maintient sans fluctuations importantes jusqu'au terme de $\mathrm{PS}_{2}$.

Chez les individus isolés, la répartition de la présence des poissons au niveau supérieur est totalement indifférenciée de $\mathrm{AS}$ à $\mathrm{PS}_{1}$, de $\mathrm{PS}_{1}$ à $\mathrm{PS}_{2}$ et de $\mathrm{AS}$ à $\mathrm{PS}_{2}$. Au niveau inférieur, l'évolution est significative dans tous les cas; elle s'accentue très légèrement de $\mathrm{PS}_{1}$ à $\mathrm{PS}_{2}$ et se maintient au cours de $\mathrm{PS}_{2}$ sans revenir à la situation initiale observée en $\mathrm{AS}$. Ces comparaisons indiquent une répartition maximale et continue au niveau inférieur après l'intervention du stimulus.

Les contrôles statistiques confirment le fait qu'en groupe, les Caecobarbus nourris antérieurement et stimulés avec de la nourriture flottante tendent à gagner la surface après l'intervention du stimulus. La différence est significative de AS à $\mathrm{PS}_{1}$, pour le niveau supérieur, mais non pour le niveau inférieur. Leur présence y est de courte durée en sorte que de $\mathrm{PS}_{1}$ à $\mathrm{PS}_{2}$ les modifications sont significatives. Entre AS et $\mathrm{PS}_{2}$, la différence n'est pas marquante, ce qui signifie que les poissons rétablissent rapidement leur répartition verticale initiale.

Chez les individus isolés, la présence au niveau supérieur après stimulation est plus longue. Cette situation se maintient jusqu'en $\mathrm{PS}_{2}$, avec tendance significative à revenir à la répartition initiale sur le fond comme en AS.

\section{Tableau 14}

Evolution des réactions de $C$. geertsi au cours de AS, $\mathrm{PS}_{1}$ et $\mathrm{PS}_{2}$. Poissons nourris antérieurement et stimulés avec de la nourriture flottante.

\begin{tabular}{llcc}
\multicolumn{2}{l}{$\begin{array}{l}\text { Périodes } \\
\text { comparées }\end{array}$} & $\begin{array}{c}\mathrm{P} \\
\text { (Poissons en groupe) }\end{array}$ & $\begin{array}{c}\mathrm{P} \\
\text { (poissons isolés) }\end{array}$ \\
\cline { 3 - 4 } $\mathrm{AS}_{\mathrm{S}}$ & $\mathrm{PS}_{1 \mathrm{~s}}$ & 0,029 & 0,014 \\
$\mathrm{AS}_{\mathrm{i}}$ & $\mathrm{PS}_{1 \mathrm{i}}$ & 0,100 & 0,014 \\
$\mathrm{PS}_{1 \mathrm{~s}}$ & $\mathrm{PS}_{2 \mathrm{~s}}$ & 0,029 & 0,171 \\
$\mathrm{PS}_{1 \mathrm{i}}$ & $\mathrm{PS}_{2 \mathrm{i}}$ & 0,014 & 0,014 \\
$\mathrm{AS}_{\mathrm{S}}$ & $\mathrm{PS}_{2 \mathrm{~s}}$ & 0,100 & 0,171 \\
$\mathrm{AS}_{\mathrm{i}}$ & $\mathrm{PS}_{2 \mathrm{i}}$ & 0,171 & 0,014 \\
\hline
\end{tabular}

Lorsque l'on compare ces résultats (Tableau 14) avec ceux obtenus sur Anoptichthys (Tableau 7), on constate une fois de plus que la tendance à se maintenir au niveau inférieur est sensiblement plus marquée chez les Caecobarbus, même lorsque le stimulus est présenté en surface. Ce phénomène est plus marqué chez les poissons observés en groupe et a pour effet de favoriser un rétablissement de la situation initiale dès la fin de $\mathrm{PS}_{1}$. 
Comme chez les Anoptichthys, la stimulation par un choc mécanique exerce sur la distribution verticale des Caecobarbus d'une periode à la suivante, des effets très différents de ceux qui résultent de la stimulation chimique. Chez les poissons en groupe nourris antérieurement avec des Tubifex, les deux seules différences significatives concernent le niveau supérieur: la densité qu'on y observe se réduit notablement de $\mathrm{AS}$ à $\mathrm{PS}_{1}$ et $\mathrm{PS}_{2}$. Contrairement à ce qui se passe chez les Anoptichthys, les niveaux inférieurs ne subissent dans ces conditions, aucune variations notable de densité au cours des trois périodes d'observation. Chez les Caecobarbus isolés comme chez les Anoptichthys la stimulation mécanique entraîne une réduction significative de la présence au niveau supérieur au cours de $\mathrm{PS}_{1}$. Mais contrairement aux Anoptichthys, cet effet s'accompagne chez les Caecobarbus isolés, d'un accroissement significatif de la présence au niveau inférieur au cours de la même période. Autre différence par rapport aux Anoptichthys isolés, l'évolution des Caecobarbus isolés est la même que celle des poissons observés en groupe de AS à $\mathrm{PS}_{2}$.

On voit que, malgré de nettes différences par rapport aux effets de la stimulation chimique, les effets de la stimulation mécanique indiquent chez les Caecobarbus une réaction préférentielle pour la zone du subtrat plus accusée que chez les Anoptichthys.

\section{Tableau 15}

Evolution des réactions de $C$. geertsi au cours de $\mathrm{AS}, \mathrm{PS}_{1}$ et $\mathrm{PS}_{2}$. Poissons nourris antérieurement avec des Tubifex et stimulés par un choc mécanique.

\begin{tabular}{lccc}
\hline \multicolumn{2}{l}{\begin{tabular}{l} 
Périodes \\
\multicolumn{2}{l}{ Comparées }
\end{tabular}} & $\begin{array}{c}\mathrm{P} \\
\text { (Poissons en groupe) }\end{array}$ & $\begin{array}{c}\mathrm{P} \\
\text { (Poissons isolés) }\end{array}$ \\
$\mathrm{AS}_{\mathrm{S}}$ & $\mathrm{PS}_{1 \mathrm{~s}}$ & 0,014 & 0,014 \\
$\mathrm{AS}_{\mathrm{i}}$ & $\mathrm{PS}_{1 \mathrm{i}}$ & 0,057 & 0,029 \\
$\mathrm{PS}_{1 \mathrm{~s}}$ & $\mathrm{PS}_{2 \mathrm{~s}}$ & Pas de différence & Pas de différence \\
$\mathrm{PS}_{1 \mathrm{i}}$ & $\mathrm{PS}_{2 \mathrm{i}}$ & 0,100 & 0,443 \\
$\mathrm{AS}_{\mathrm{S}}$ & $\mathrm{PS}_{2 \mathrm{~s}}$ & 0,014 & 0,014 \\
$\mathrm{AS}_{\mathrm{i}}$ & $\mathrm{PS}_{2 \mathrm{i}}$ & 0,171 & 0,057 \\
\hline
\end{tabular}

Chez les poissons en groupe nourris antérieurement avec de la nourriture flottante, la stimulation mécanique n'entraîne aucune évolution typique de densité d'une période à l'autre pour les deux niveaux considerés. Ches les Caecobarbus isolés et nourris antérieurement avec de la nourriture flottante, l'évolution de AS à $\mathrm{PS}_{1}$ est à l'opposé de celle trouvée chez les Anoptichthys isolés: les poissons se portent significativement au niveau inférieur. Cette tendance s'inverse au cours de $\mathrm{PS}_{2}$, bien que la comparaison de AS et de $\mathrm{PS}_{2}$ indique un accroissement de la présence des poissons au niveau inférieur au cours de la dernière période. 
Evolution des réactions de $C$. geertsi au cours de $\mathrm{AS}, \mathrm{PS}_{1}$ et $\mathrm{PS}_{2}$. Poissons nourris antérieurement evec de la nourriture flottante et stimulés par un choc mécanique.

\begin{tabular}{llcc}
\hline \multicolumn{2}{l}{$\begin{array}{l}\text { Périodes } \\
\text { comparées }\end{array}$} & $\begin{array}{c}\mathrm{P} \\
\text { (Poissons en groupe) }\end{array}$ & $\begin{array}{c}\mathrm{P} \\
\text { (Poissons isolés) }\end{array}$ \\
$\mathrm{AS}_{\mathrm{S}}$ & $\mathrm{PS}_{1 \mathrm{~s}}$ & 0,171 & 0,014 \\
$\mathrm{AS}_{\mathrm{i}}$ & $\mathrm{PS}_{1 \mathrm{i}}$ & 0,171 & 0,014 \\
$\mathrm{PS}_{1 \mathrm{~s}}$ & $\mathrm{PS}_{2 \mathrm{~s}}$ & 0,100 & Pas de différence \\
$\mathrm{PS}_{1 \mathrm{i}}$ & $\mathrm{PS}_{2 \mathrm{i}}$ & 0,343 & 0,014 \\
$\mathrm{AS}_{\mathrm{S}}$ & $\mathrm{PS}_{2 \mathrm{~s}}$ & 0,243 & 0,029 \\
$\mathrm{AS}_{\mathrm{i}}$ & $\mathrm{PS}_{2 \mathrm{i}}$ & 0,443 & 0,029 \\
\hline
\end{tabular}

\section{DISCUSSION ET CONCLUSIONS}

1. Le phénomène le plus important que nos expériences mettent en évidence est la modification systématique de l'orientation verticale de la nage en fonction du type de nourriture utilisé. Les expériences définitives ayant été effectuées au terme d'une période de 8 jours, au cours de laquelle des groupes distincts de poissons avaient été nourris de façon exclusive, soit avec de la nourriture gagnant le substrat par gravité, soit avec de la nourriture flottante, les effects constatés résultent évidemment de l'apprentissage. En ce qui concerne l'Anoptichthys, deux remarques doivent être faites à ce propos. En premier lieu, la tendance de ce poisson à plonger vers le substrat après l'intervention d'une stimulation chimique ou d'une stimulation mécanique est très accusée dès le stade juvénile (Thinès, Soffié et Vandenbussche, 1966). Sans exclure l'influence que peut exercer l'utilisation systématique d'une nourriture plongeante (Tubifex) au cours de l'élevage, en particulier aux stades précoces du développement, toutes nos observations antérieures, tant sur les alevins que sur les adultes, semblaient indiquer que l'on avait affaire à un stéréotype spécifique. En second lieu, il est certain que la capacité d'apprentissage est plus développée chez Anoptichthys (Thinès et Kähling, 1957) que chez Caecobarbus, le premier pouvant être dressé de façon systématique, tandis que les essais de conditionnement ont toujours échoué chez le second, au moins lorsqu'on utilise un stimulus lumineux comme excitant conditionné.

2. L'utilisation de l'un ou de l'autre type de nourriture exerce des effets qui se traduisent par une modification sensible de l'orientation de la nage, soit vers le substrat, soit vers la surface. Avant l'intervention du stimulus, les niveaux préférentiels sont le niveau inférieur et le niveau moyen, tant ches les Anop- 
tichthys que chez les Caecobarbus, lorsqu'ils ont été nourris antérieurement avec de la nourriture plongeante. Chez les Caecobarbus toutefois, l'utilisation antérieure de nourriture flottante ne détermine, avant le stimulus, aucune tendance nette à visiter le niveau supérieur. Cette observation concorde avec ce qui a été dit plus haut au sujet de la capacité d'apprentissage plus faible du Caecobarbus. Il semble que ce poisson modifie plus difficilement que l'Anoptichthys l'orientation fondamentale de sa locomotion.Ceci, joint aux résultats de nos observations antérieures, permet de supposer que cette orientation verticale est un stéréotype qui, sans être spécifique d'une espèce particulière, serait présent à des degrés divers non seulement chez les deux formes étudiées, mais aussi chez d'autres poissons cavernicoles.

3. Chez l'Anoptichthys, la nage orientée vers la zone où se trouve la nourriture est suivie d'une exploration active qui aboutit à la saisie de particules nutritives. Chez le Caecobarbus, nous l'avons signalé, ce processus est plus lent et moins précis. Dans les expériences où l'on utilisait un stimulus mécanique, on observe chez le Caecobarbus une nette dissociation entre l'accélération initiale de la nage après la stimulation et l'exploration de la zone où la nourriture est présente. Si l'on rapproche de ce phénomène le fait que, avant l'intervention du stimulus, les Caecobarbus manifestent une préférence pour le niveau inférieur même s'ils ont été nourris antérieurement en surface, on peut se demander si la tendance à se porter vers le substrat n'est pas, au moins chez cette espèce, un stéréotype autonome, indépendant, en soi, de l'exploration alimentaire.

4. Si un tel stéréotype comportemental existe, il se révèle néanmoins relativement labile, ainsi que l'établissent les résultats obtenus sur les poissons nourris antérieurement en surface. Les effets constatés ayant été obtenus après un traitement de 8 jours aux deux niveaux verticaux extrêmes, il y a lieu de rapprocher ceux-ci des observations de Heuts et Thinès (1971) sur les Barbus conchonius aveuglés. Bien que, dans ce dernier cas, une période beaucoup plus longue ( 8 mois) se fût écoulée entre l'aveuglement des animaux et les observations, la modification comportementale se traduit, ici encore, par un changement du niveau préférentiel de la locomotion. Chez les Barbus aveuglés comme chez les deux formes cavernicoles étudiées, on constate une inversion de l'orientation de la nage. Celle-ci est spontanée chez les Barbus et acquise chez les cavernicoles. L'ensemble de ces faits permet de conclure à l'existence d'une polarisation verticale de la locomotion, axée principalement sur le substrat.

5. L'action spécifique de la stimulation chimique apparaît clairement lorsque l'on compare ses effets à ceux de la stimulation mécanique. Ces derniers provoquent des réactions nettement moins orientées, dans lesquelles la composante phobique entraîne une indifférenciation notable dans l'établissement du niveau préférentiel. L'autonomie supposée du stéréotype chez Caecobarbus se manifeste, ici encore, dans le fait que les poissons ont tendance, même dans ces conditions, à gagner le substrat après la stimulation, quel que soit le type de nourriture utilisé antérieurement. Chez les Anoptichthys, au contraire, la fuite est orientée vers le substrat ou vers la surface selon que les animaux ont été nourris antérieurement avec de la nourriture plongeante ou avec de la nourriture flottante. 
6. Les faits discutés au point précédent suggèrent une interprétation en relation avec l'ancienneté phylogénique. Si, en conformité avec la catégorisation proposée par Vandel (1964), on considère qu'Anoptichthys est un cavernicole récent et Caecobarbus un cavernicole ancien, on peut supposer que la variabilité des réactions est plus marquée chez le premier que chez le second. La fixité relative du stéréotype chez le Caecobarbus entrainerait une polarisation sur le substrat qui serait plus résistance à l'action d'influences survenant dans le cours de l'ontogenèse (apprentissages) et expliquerait la réapparition de la réaction préférentielle pour le niveau inférieur dans des conditions contrecarrant celle-ci. De même, la dissociation entre polarisation et exploration alimentaire pourrait être mise en relation avec des processus de dégénérescence dans les intégration comportementales, elles-mêmes liées à l'ancienneté phylétique.

7. Un aspect important de nos expériences concerne les différences éventuelles entre les résultats obtenus sur les poissons en groupe et sur les poissons isolés. Chez les Anoptichthys, il existe une différence significative au cours de AS entre les poissons nourris antérieurement avec des Tubifex et les poissons nourris avec des Daphnies lorsqu'ils sont en groupe, le niveau préférentiel étant fonction du type de nourriture $(P=0,014)$. Cette différence ne se retrouve pas chez les poissons isolés. Le même phénomène s'observe chez les poissons isolés nourris antérieurement avec des Daphnies. Les poissons en groupe et les poissons isolés réagissent de la même façon à la nourriture plongeante et à la stimulation mécanique lorsqu'ils ont été nourris antérieurement avec des Tubifex. Les réactions à la nourriture flottante et à la stimulation mécanique sont identiques chez les poissons en groupe et chez les poissons isolés nourris antérieurement les uns et les autres avec des Daphnies, au cours de $\mathrm{PS}_{1}$ seulement $(\mathrm{P}=0,014)$. Le fait d'être en groupe semble donc faciliter avant tout, chez les Anoptichthys la réaction préférentielle pour le niveau auquel la nourriture est présentée.

Chez les Caecobarbus, l'influence du groupe est peu définie. L'influence du type de nourriture sur le choix du niveau préférentiel n'est déterminante que chez les poissons isolés en AS. La même conclusion s'impose lorsque l'on compare les réactions à la stimulation mécanique des poissons nourris antérieurement avec des Tubifex à celles des poissons nourris antérieurement avec des Daphnies. Dans le cas des poissons nourris antérieurement avec des Tubifex, les poissons isolés présentent une nette réaction préférentielle pour le niveau inférieur lorsqu'on les stimule avec de la nourriture plongeante, cette réaction allant à l'inverse de celle que l'on observe lorsque l'on utilise la stimulation mécanique. Dans les mêmes conditions, les réactions des poissons observés en groupe sont totalement indifférenciées. Dans le cas des poissons nourris antérieurement avec de la nourriture flottante, les poissons isolés réagissent significativement à l'excitation chimique, par opposition à l'excitation mécanique, dans l'établissement de leur niveau préférentiel en $\mathrm{AS}$ et en $\mathrm{PS}_{2}$. Les Caecobarbus en groupe ne présentent pas cette différenciation dans les mêmes conditions.

Il apparaît donc que chez les Caecobarbus, le fait de nager en groupe soit plutôt inhibiteur. Rappelons toutefois que les groupes de Caecobarbus observés étaient numériquement moins importants que les groupes d'Anoptichthys. Les 
données comparatives manquent pour établir si la forme cavernicole a subi, sous le rapport des comportements de groupe, une dégénérescence à partir de structures qui autaient été présentes chez la forme ancestrale épigée.

8. Quelques remarques doivent encore être faites au sujet de la détection des proies. L'utilisation de proies plongeantes et de proies flottantes détermine en effet deux catégories de stimulation qui différent, non seulement quant à la position verticale qu'elles occupent dans l'aquarium expérimental, mais peut-être aussi quant à la nature de l'information sensorielle qu'elles transmettent.

Toutes les expériences ont été effectuées en évitant soigneusement les perturbations mécaniques lors de la présentation des stimuli. Néanmoins, il est certain que la descente des Tubifex vers le substrat produit une stimulation mécanique en même temps qu'une stimulation chimique. Dans le cas de la nourriture flottante, il est probable que la présence des Daphnies en surface est détectée mécaniquement avant d'être perçue chimiquement. Celles-ci en effet, contrairement aux Tubifex, sont sèches et ne permettent aucune diffusion dans la mase liquide.

Tout en constituant une garantie de différenciation spatiale entre les deux niveaux comparés, les caractéristiques respectives des deux catégories de nourriture doivent être prises en considération dans le déclenchement de l'accélération locomotrice qui suit immédiatement la présentation du stimulus. Ultérieurement, lorsque les poissons sont arrivés à proximité des proies, la stimulation chimique intervient, tant dans le cas de la nourritura flottante que dans le cas de la nourriture plongeante, en sorte que les situations sont identiques à partir de ce moment.

De toute manière, les expériences montrent que les deux types de nourriture utilisée agissent efficacement pour déterminer l'orientation de la nage vers le substrat ou vers la surface après un certain temps d'apprentissage de la localisation de l'un ou l'autre type de proie. Ajoutons que l'intervention éventuelle de la stimulation mécanique dans la détection de la nourriture ne constitue pas un facteur perturbant; les expériences dans lesquelles on utilisait exclusivement ce type de stimulation, ont en effet montré que, même si les résultats obtenus dans ces conditions diffèrent de ceux que l'on obtient avec la nourriture, celles-ci n'empêchent pas l'établissement d'un niveau vertical préférentiel.

\section{RESUME}

Des expériences systématiques effectuées sur les poissons cavernicoles Anoptichthys jordani et Caecobarbus geertsi montrent qu'il est possible d'obtenir une modification significative de l'orientation verticale de la nage, en nourrisant les animaux pendant les 8 jours précédant les observations définitives, soit avec de la nourriture plongeante, soit avec de la nourriture flottante.

Les observations ont été quantifiées en relevant les positions des poissons en hauteur dans un aquarium divisé en 3 niveaux d'observation. Ces relevés étaient 
effectués toutes les 10 secondes pendant une durée de 15 minutes, divisée en trois périodes de 5 minutes [Minutes 0 à 5 : avant stimulation (AS); minutes 5 à 10 : première période après la stimulation $\left(\mathrm{PS}_{1}\right)$; minutes 10 à 15 : seconde période après stimulation $\left.\left(\mathrm{PS}_{2}\right)\right]$. Chaque type d'observation a été répété 4 fois à des intervalles de plusieurs jours. Les moyennes des observations par période de 5 minutes ont été comparés en utilisant l'épreuve U de Mann-Whitney $(\alpha=0,05)$.

Les modifications d'orientation dues à la nourriture flottante montrent que le stéréotype de polarisation sur le substrat, qui existe chez Anoptichthys et, avec de légères différences, chez Caecobarbus, est un caractère comportemental relativement labile. Cette labilité est plus accusée chez Anoptichthys que chez Caecobarbus, phénomène que l'on peut théoriquement interpréter à partir du fait que le Caecobarbus est un cavernicole plus ancien que l'Anoptichthys. Le stéréotype de polarisation sur le substrat serait, dans cette hypothèse, plus modifiable chez l'Anoptichthys en raison d'une variabilité des montages comportementaux, laquelle serait moindre chez une forme plus ancienne comme Caecobarbus. Dans un même ordre d'idées, on remarque que le traitement systématique à la nourriture flottante ne détermine pas de réaction préférentielle pour le niveau supérieur avant la stimulation chimique chez Caecobarbus, le phénomène inverse s'observant chez Anoptichthys. La stimulation mécanique induit des réactions phobiques dont l'orientation verticale dominante dépend, chez Anoptichthys, du type de nourriture utilisé au cours du traitement préalable. Ce phénomène ne s'observe pas chez Caecobarbus, lequel manifeste dans ces conditions une réaction préférentielle pour le substrat.

Il est remarquable que, malgré le rigidité plus accusée de la polarisation sur le substrat chez Caecobarbus, l'orientation préférentielle vers ce niveau ne soit pas suivie d'une exploration systématique. Chez Anoptichthys au contraire, l'orientation polarisée est toujours suivie d'exploration. La dissociation de la polarisation et de l'exploration constituent un indice de dégénérescence phylétique plus marqué chez le Caecobarbus sur le plan éthologique. L'influence du groupe semble favoriser chez l'Anoptichthys, la réaction préférentielle pour le niveau auquel la nourriture est présentée. Chez le Caecobarbus, la présence de congénères est plutôt inhibitrice.

\section{SUMMARY}

Systematic experiments on the cave-fishes Anoptichthys jordani and Caecobarbus geertsi show that the vertical orientation of swimming behaviour can be significantly modified by feeding the fishes during a period of 8 days before the experiments, either with food dropping to the bottom (Tubifex) or with food floating on the surface of the water (dried Daphnia).

The observations have been quantified by recording systematically the position of the fishes in height in an aquarium divided in 3 levels of observation. The position of the fishes was recorded every 10 seconds during a total period of 15 minutes, divided in 3 periods of 5 minutes each [Minutes 0 to 5: anterior to stimulation (AS); Minutes 5 to 10 : first period after stimulation $\left(\mathrm{PS}_{1}\right)$; Minutes 
10-15: second period after stimulation $\left(\mathrm{PS}_{2}\right)$ ]. Each type of observation has been repeated 4 times at intervals of several days. The median values of the 5 minutes observation periods have been compared by means of the Mann-Whitney U test $(\alpha=0,05)$.

The orientation changes resulting from systematic feeding with floating food, show that the stereotype of polarisation on the substrate, which exists in Anoptichthys and, with some differences, in Caecobarbus, is a rather variable behavioural character. This movement is more pronounced in Anoptichthys than in Caecobarbus. This difference can be theoretically interpreted in relation with the fact that Caecobarbus is a more ancient cave-form than Anoptichthys. According to this hypothesis, the stereotype of polarisation on the substrate would be more liable to be modified in Anoptichthys, due to a higher variability of behaviour structures, the latter being more rigid in an ancient form in relation with phyletic degeneration processes typical of true troglobiotic animals. Similarly, systematic feeding with floating material does not cause any preferential reaction for the upper water level before chemical stimulation in Caecobarbus, the reverse being true for Anoptichthys. Mechanical stimulation induces phobic reactions whose dominant vertical orientation is determined, in Anoptichthys, by the type of feeding material used during the pre-experimental period. This does not hold for Caecobarbus, who shows a definite preference for the substrate in these conditions.

It is remarkable that, in spite of the more rigid polarisation on the substrate shown by Caecobarbus, the preferential orientation to the lower level is not followed by an active exploratory behaviour as in Anoptichthys. This dissociation between substrate polarisation and exploratory behaviour is to be interpreted, once more, as a sign of deeper phyletic degeneration in the ethology of Caecobarbus. In Anoptichthys the effects of the group seem to favour the preferential reaction for the vertical level at which food is present, whereas in Caecobarbus, the presence of specific mates is rather inhibitory. 


\section{BIBLIOGRAPHIE}

GLASER, D., 1968 - Zum Verhalten blinder Fische. Z. Tierpsychol. 25: 648-658.

LÜLING, K.H., 1954 - Untersuchungen am Blindfisch Anoptichthys jordani Hubbs und Innes (Characidae). - II. Beobachtungen und Experimente an Anoptichthys jordani zur Prüfung der Einstellung zum Futter und zur Wasserturbulenz. Zool. Jahrb. (Abt. Zool.), 65: 9-42.

HEUTS, B. and THINESS, G., 1971 - Behavioural Changes in a Stock of Barbus conchonius (Hamilton, Pisces, Cyprinidae) after Accidentally induced partial Depigmentation and Blindness. Z. Tierpsychol., 28: 113-163.

THINÈS, G. et CAPON, D., 1971 - Effets de la suppression du contrôle visual sur le comportement alimentaire de Barbus conchonius et de Danio malabaricus (Pisces, Cyprinidae) (En préparation).

THINÈS, G. und KÄHLING, J., 1957 - Untersuchungen über die Farbempfindlichkeit des blinden Höhlenfisches Anoptichthys jordani Hubbs und Innes (Characidae). Z. Biol., 109: 150-160.

THINÈS, G., SOFFIÉ, M. et VANDENBUSSCHE, E., 1966 - Analyse du comportement alimentaire du poisson cavernicole aveugle Anoptichthys Gen. et d'hybrides $\mathrm{F}_{1}$ (Astyanax X Anoptichthys) et $\mathrm{F}_{2}$. Int. J. Speleol., 2: 437-448.

VANDEL, A., 1964 - Biospéléologie. La Biologie des Animaux Cavernicoles Paris, GauthierVillars. 